\title{
Monitoring activity in neural circuits with genetically encoded indicators
}

\author{
Gerard J. Broussard ${ }^{1,2}$, Ruqiang Liang ${ }^{1}$ and Lin Tian ${ }^{1,2}$ * \\ ' Department of Biochemistry and Molecular Medicine, University of California Davis, Davis, CA, USA \\ ${ }_{2}^{2}$ Neuroscience Graduate Group, University of California Davis, Davis, CA, USA
}

\section{Edited by:}

Katsuhiko Mikoshiba, RIKEN Brain

Science Institute, Japan

\section{Reviewed by:}

Mazahir T. Hasan,

Charité-Universitätsmedizin-Berlin, Germany

Bernd Kuhn, Okinawa Institute of Science and Technology Graduate University, Japan

\section{${ }^{*}$ Correspondence:}

Lin Tian, Department of Biochemistry and Molecular Medicine, University of California Davis, Davis, CA 95817, USA

e-mail: lintian@ucdavis.edu
Recent developments in genetically encoded indicators of neural activity (GINAs) have greatly advanced the field of systems neuroscience. As they are encoded by DNA, GINAs can be targeted to genetically defined cellular populations. Combined with fluorescence microscopy, most notably multi-photon imaging, GINAs allow chronic simultaneous optical recordings from large populations of neurons or glial cells in awake, behaving mammals, particularly rodents. This large-scale recording of neural activity at multiple temporal and spatial scales has greatly advanced our understanding of the dynamics of neural circuitry underlying behavior-a critical first step toward understanding the complexities of brain function, such as sensorimotor integration and learning. Here, we summarize the recent development and applications of the major classes of GINAs. In particular, we take an indepth look at the design of available GINA families with a particular focus on genetically encoded calcium indicators (GCaMPs), sensors probing synaptic activity, and genetically encoded voltage indicators. Using the family of the GCaMP as an example, we review established sensor optimization pipelines. We also discuss practical considerations for end users of GINAs about experimental methods including approaches for gene delivery, imaging system requirements, and data analysis techniques. With the growing toolbox of GINAs and with new microscopy techniques pushing beyond their current limits, the age of light can finally achieve the goal of broad and dense sampling of neuronal activity across time and brain structures to obtain a dynamic picture of brain function.

Keywords: in vivo imaging, mammalian and rodent brain, neural activity, genetically encoded sensors, calcium sensor, voltage sensor, synaptic activity reporter

\section{INTRODUCTION}

Within the mammalian brain, neuronal and glial cells communicate at spatial and temporal scales spanning orders of magnitude. One of the fundamental challenges with which modern neuroscience is currently grappling is the development of tools that can record this communication as it occurs at the relevant scales. Furthermore, it is desirable that these tools should be deployable in living animal models. Such probes will aid the study of neural communication within the context of phenomena such as experience dependent plasticity, sensorimotor integration, learning, and memory.

An extensive set of tools for studying brain function in vivo currently exists, and each of these possesses its own set of strengths and weaknesses. For example, recordings of intracellular voltage or transmembrane current can be made using patch clamp (Liu et al., 2009; Crochet et al., 2011) or extracellular potential recordings can be achieved with fine-tipped metallic electrodes (Hubel and Wiesel, 1959; Reid et al., 1997; Mante et al., 2013). These methods provide an exquisitely detailed temporal signal, but are limited by the number of cells that can be recorded simultaneously. Simultaneous neural recordings with near-cellular resolution can be achieved using multiple electrode arrays (Buzsaki, 2004). However, this technique cannot precisely localize cell position and yields limited information about cell types. Optical imaging of bolus loaded AM ester dyes allows recordings at high spatial resolution from many cells simultaneously (Stosiek et al., 2003; Helmchen and Denk, 2005). But this technique results in high background due to residual fluorescence in the extracellular space as well as the lack of genetic control (Stosiek et al., 2003). A lack of genetic control can in particular lead to increased fluorescent background due to unspecific staining of non-neuronal cells as well as neurons not pertinent to a given study. In addition, small molecule-based dyes are not compatible with chronic imaging (Aramuni and Griesbeck, 2013). Both small-molecule dyes and electrode methods are highly invasive and can negatively impact the health of recorded cells (Polikov et al., 2005; Dombeck et al., 2010) making chronic recordings from the same cell population difficult.

Genetically encoded indicators combined with modern microscopy (such as multi-photon microscopy), can potentially overcome these challenges to allow non-invasive, ultrasensitive and chronic measurement at specific synapses and within or across circuit elements in behaving animals. A large array of protein based indicators has been created to monitor neurotransmission, synaptic spillover, excitable membrane potential, calcium dynamics, vesicle trafficking, receptor mobilization and other essential biochemical events related to neural activity. A subset of these-detecting changes in intracellular calcium concentration, synaptic signaling events, and changes in membrane 
potential-have been successfully deployed for the imaging of action potentials (APs) in vivo. Here, we refer to this group of probes as genetically encoded indicators of neural activity (GINAs). For a more exhaustive review of available fluorescent sensors of cellular activity, please see VanEngelenburg and Palmer (2008), Ibraheem and Campbell (2010). Application of GINAs have facilitated large-scale recording of neural activity in genetically identified populations over multiple spatial and time scales in living neurons in vitro, ex vivo, and in vivo.

Here we review advances in design and engineering of GINAs. We discuss various properties of genetically encoded calcium indicators (GECIs) and their optimization for improved detection of single and multiple APs in in vivo imaging. In particular, as a case study, we present protein engineering efforts to incrementally improve intrinsic properties of the GCaMP family to match with challenging signal to noise ratio (SNR) in in vivo imaging. We further discuss technologies developed to aid in vivo imaging in the rodent brain. Finally, we briefly summarize recent findings based on the strength of GINAs as a toolbox for analyzing neural circuit function.

\section{DESIGN OF GENETICALLY ENCODED INDICATORS OF NEURAL ACTIVITY}

The first protein used to detect functionally relevant changes within a cell was an aequorin protein which was purified from jellyfish (Shimomura et al., 1962) and introduced exogenously to muscle fibers of a barnacle to sense changes to intracellular calcium that occur during fiber contraction (Ashley and Ridgway, 1968). The era of the modern GINAs, however, began in earnest with the introduction of biosensors based on fluorescent proteins (FPs) which were able to detect changes to intracellular calcium levels (Miyawaki et al., 1997; Romoser et al., 1997), membrane voltage (Siegel and Isacoff, 1997) and synaptic vesicle secretion (Miesenbock et al., 1998). A guide to the general design of several GINA families is presented in Figure 1.

Genetically encoded indicators of neural activity typically consist of an analyte-binding or sensing domain and a reporter element which is based on either a single FP or two FPs. In the case of single FP GINAs, changes in the cellular environment detected by the analyte-binding or sensing domain result in changes in the chromophore environment of the FP leading to an increment or decrement of fluorescence intensity (Siegel and Isacoff, 1997; Baird et al., 1999; Nakai et al., 2001). For example, as illustrated diagrammatically in Figure 1A, in the scaffold of GCaMPs, calmodulin (CaM) binds the M13 peptide from myosin light chain kinase in the presence of calcium; this coupling reverses when calcium is absent (Nakai et al., 2001). The sensor domain transduces conformational changes of analyte binding to a change in the fluorescence intensity through its coupling with the reporter $\mathrm{FP}(\mathrm{s})$. Additionally, some single FP-based GINAs contain FPs in which fluorescence intensity is environmentally sensitive (e.g., $\mathrm{pH}$ sensitive FPs; Figure 1D; Miesenbock et al., 1998). In the case of two FP based sensors, the conformational changes in the analytebinding or sensing domain lead to Förster resonance energy transfer (FRET) between two FPs with overlapping excitation and emission spectra. For example, the Cameleon family contains both M13 and CaM in between a blue/green or cyan/yellow FP pairs as shown for Yellow Cameleon-Nano (YC-Nano; Figure 1B). Upon calcium binding, the relative distance and orientation of the FPs are altered, resulting in a change in the non-radiative transfer of energy between the donor and acceptor chromophores.

Single-FP based GINAs are prone to motion artifacts, which can be corrected by algorithms (e.g., Dombeck et al., 2007) and using a reference fluorophore. On the other hand, the FRET based GINAs, by virtue of ratiometric imaging can cancel the motionrelated artifacts (Wallace et al., 2008; Lütcke et al., 2010) and are thus highly suitable for imaging neuronal activity in freely moving animals. However, the SNR of FRET-based probes are lower than their single FP counterparts in probing higher numbers of APs (see, e.g., Tian et al., 2009). Single-FP based probes also preserve spectral bandwidth for applications in multiplex imaging and optogenetics. Although the currently used FRET sensors can be coupled with red-shifted FPs and optogenetic tools, the broad emission and excitation spectra of FRET sensors reduce some flexibility.

Relative to other methods for recording neural signals, GINAs possess several advantages. First, they can be targeted to genetically defined cell populations of interest. As such, they can be introduced via minimally invasive interventions such as viral mediated gene delivery or transgenesis (permanent genomic modification). Genetic control also reduces background signal from sources not under scrutiny, increasing SNR (Stosiek et al., 2003), and can permit targeting to a population that is anatomically or functionally related. For example, GINAs allow for specific labeling of direct and indirect pathway neurons in the striatum (Cui etal., 2013) as well as excitatory (Bozza et al., 2004; Ziv etal., 2013) and inhibitory (Kaifosh etal., 2013) neurons which co-localize within the same local neural circuit. Second, GINAs make it possible to record from a large population of cells simultaneously with better spatial resolution than the best electrophysiological techniques (Knöpfel, 2012). Studies exploiting this strength have demonstrated neural network characteristics that were not previously apparent from single cell recordings (Dombeck et al., 2010; Harvey et al., 2012). Finally, GINAs allow for chronic interrogation of the same cells for long periods of time (Mank et al., 2008; Tian et al., 2009; Kuhn et al., 2012; Margolis et al., 2012). As a result, studies which track dynamics of neural circuitry such as learning and memory induced changes to neural ensembles have been made possible (Huber et al., 2012; Petreanu et al., 2012).

Used in conjunction with modern microscopic techniquesmost notably two photon (2P) imaging-GINAs are now routinely employed in the interrogation of neural activity under a wide variety of contexts in awake, behaving mammals (Helmchen and Denk, 2005; Bovetti et al., 2014).

\section{GENETICALLY ENCODED CALCIUM INDICATORS}

Calcium dynamics are a proxy to monitor APs in neurons (Tank et al., 1988; Miyawaki et al., 1997) and represent excitable states in astrocytes (Perea and Araque, 2005). In neurons, APs lead to calcium transients in the cytosol through voltage-gated calcium channels (Hille, 2001). This rise is reversed as calcium is buffered, extruded, and pumped back into internal stores 

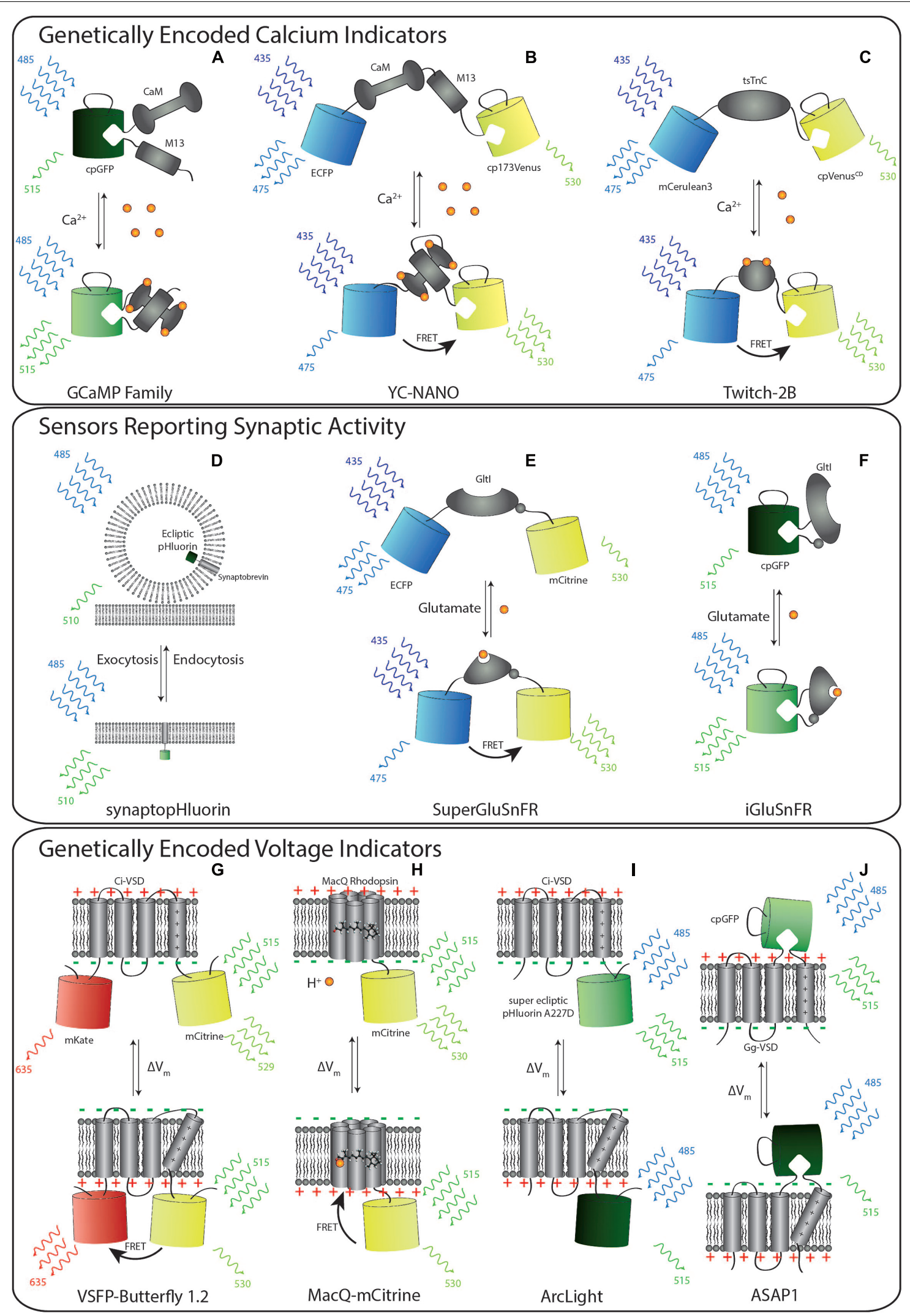

FIGURE 1 | Continued 


\section{FIGURE 1 | Continued}

Schematic representation of selected members of genetically encoded indicators of neural activity (GINA). (A-C) Calcium indicators.

(A) GCaMP6 consists of circularly permuted green fluorescent protein (cpGFP) inserted between calmodulin (CaM) and an M13 peptide. Upon calcium binding, conformational changes in the CaM-M13 complex induce fluorescence changes in the circularly permuted enhanced green fluorescent protein (cpEGFP). (B) YC-Nano contains CaM-M13 complex sandwiched between the donor FP (ECFP) to the acceptor FP (cp173Venus). Upon calcium binding, conformational changes of CaM-M13 complex increases Förster resonance energy transfer (FRET) between the FP pair. The result is an increase in the ratio of the fluorescence in the yellow to blue channels. (C) Likewise, Twitch-2B increases FRET upon calcium being bound by its calcium sensitive domain, tsTnC. (D-F) GINAs reporting synaptic activity. (D) SynaptopHluorin consists of a ecliptic pHluorin FP fused to the C-terminus of the vesicular protein. Vesicular release places the $\mathrm{FP}$ in the low $\mathrm{pH}$ environment of the extracellular space, which leads to the deprotonation of the fluorophore and increases pHluorin fluorescence. (E) SuperGluSnFR is a linear fusion of CFP, the Escherichia coli glutamate binding domain, Gltl, and mCitrine. Upon glutamate binding, the conformational changes of Gltl result in FRET between CFP to mCitrine. (F) iGluSnFR contains a cpGFP fused to the glutamate binding domain. The conformation changes of $\mathrm{Glt}$ induced upon glutamate binding results in deprotonation and increased fluorescence of cpGFP. (G-J) Genetically encoded voltage indicators. (G) Upon membrane depolarization, the voltage-sensing domain of Ci-VSP enters its activated state. The FPs of voltage sensitive fluorescent protein (VSFP)-Butterfly 1.2 are thus drawn into close proximity to one another, increasing FRET. (H) The opsin-based MacQ-mCitrine reduces fluorescence output upon membrane depolarization. This effect is driven by enhanced FRET from the mCitrine FP to the weakly fluorescent retinal caused by a shift in the cofactor absorption spectrum upon protonation of the Schiff base. (I) ArcLight consists of Ci-VSD and super ecliptic pHluorin carrying the point mutation A227D. Depolarization of the membrane results in a decrease in the fluorescence output of the pHluorin, but the mechanism of this change remains elusive. (J) ASAP-1 contains cpGFP fused into an extracellular linker (S3-S4 linker) of the Gag-VSP voltage sensitive domain. Depolarization quenches the fluorescence of the cpGFP.

in a process which generally lasts on the order of $100 \mathrm{~ms}$ (Helmchen etal., 1996; Koester and Sakmann, 2000). Astrocytes, a prevalent type of glial cell in the brain implicated to play important roles in synaptic plasticity, also propagate intracellular signals by means of calcium transients. The source of these calcium events in astrocytes largely overlaps those found in neurons, but due to differential expression levelsparticularly a dearth of voltage-dependent calcium channels - and relative cellular location of these sources, calcium events in these cells are longer (generally seconds in duration) and in some cases more highly localized (Ben Achour et al., 2010; Ding, 2013) or show waves of activity in other cases (Hoogland et al., 2009).

Of all GINA classes, GECIs are currently the most widely utilized for in vivo imaging in model systems including worm (Kerr et al., 2000; Boulin and Hobert, 2012), zebrafish (Higashijima et al., 2003), fly (Wang et al., 2003), rodent (Hasan et al., 2004; Tian et al., 2009), and recently non-human primate (Heider et al., 2010; Yin et al., 2014). In large part, the success of GECIs has been due to their high SNR (Mank et al., 2008; Chen et al., 2013b) via extensive protein engineering efforts to significantly improve their intrinsic properties, such as brightness (Tian et al., 2009), pH insensitivity (Miyawaki et al., 1999), stable folding (Tallini et al., 2006), photostability (Tian et al., 2009), large dynamic range (Chen et al., 2013b), fast kinetics (Sun et al., 2013) and appropriate expression level (Miyawaki et al., 1999), to match with extrinsic parameters of calcium dynamics in neurons. A summary of GECI design is shown in Figures 1A-C.

The first GECIs to gain wide usage were the FRET-based Cameleons which contain M13 and CaM in between a blue/green or cyan/yellow FP pair, conceptually similar to YC-Nano as pictured in Figure 1B. Several incrementally improved variants of Cameleon have since been engineered, e.g., the YCs series (for review, see Miyawaki, 2011; Palmer et al., 2011), the computationally redesigned variants D1cpv, D2cpv, D3cpv, and D4cpv to reduce impact on endogenous calcium handling and interaction with endogenous proteins (Palmer etal., 2006), and the highaffinity YC-Nano series (Horikawa et al., 2010). Additionally, a family of GECIs has been developed based on the muscle-specific $\mathrm{Ca}^{2++}$-binding protein Troponin C: TN-L15, TN-XL (Mank et al., 2006), TN-XXL (Mank et al., 2008), and the recent Twitch sensor (Thestrup etal., 2014). The troponin family in theory has the lowest probability of having interaction with endogenous proteins in cells. In addition, FP pairs have been optimized or replaced as newer, more advantageous FPs became available (Ai et al., 2014).

Meanwhile, a proliferation of single wavelength calcium probes (Camgaroo, Pericam, Case, and GCaMP family) based on enhanced yellow fluorescent protein (EYFP) or circular permutated green fluorescent protein (GFP) have been engineered (Baird et al., 1999; Nagai et al., 2001; Nakai et al., 2001; Souslova et al., 2007). Since then, several papers have been published on the iterative improvement of the GCaMP scaffold, which include GCaMP1.6 (Ohkura et al., 2005), GCaMP2 (Tallini et al., 2006), GCaMP3, and recently developed highly sensitive GCaMPs such as GCaMP-HS (Muto et al., 2011), Fast-GCaMPs (Sun et al., 2013) and GCaMP5 and GCaMP6 series (i.e., GCaMP6s, GCaMPm, and GCaMPf; Chen et al., 2013b). It is noted that a different group uses a distinct numbering system for GCaMP variants which include GCaMP6 (Ohkura et al., 2012), GCaMP7a (Muto et al., 2013), and GCaMP8 (Ohkura et al., 2012). However, these GCaMPs are not incremental improvements upon the GCaMP6 series (Chen et al., 2013b).

With the demonstration that a $\mathrm{YC}(\mathrm{D} 3 \mathrm{cpv})$ can readily detect a single AP in mammalian neurons, both in vitro and in vivo (Wallace et al., 2008), various members of the YC [especially YC3.60 (Nagai et al., 2004)], TN-L [especially TN-XXL (Mank et al., 2008)], and GCaMP [especially GCaMP3 (Tian et al., 2009) and GCaMP5 (Akerboom et al., 2012)] lineages have seen extensive use in in vivo preparations. Recent additions to the YC [YC-Nano (Horikawa et al., 2010)], TN-L [Twitch (Thestrup et al., 2014)], and GCaMP families [GCaMP6(s,m,f) (Chen et al., 2013b)] have achieved the long-standing goal of single spike detection in pyramidal neurons on par with or surpassing synthetic dyes in the neurons of living rodents. A summary of performance of GECIs for spike detection in in vivo mammalian preparations is presented in Table $\mathbf{1}$.

Another exciting advance in GECI development in the past few years has been the expansion of new color-palette variants, such as the red-shifted variant R-GECO (Zhao et al., 2011) based on mApple and RCaMP based on mRuby (Akerboom et al., 2013). Extending the color-spectrum has greatly increased the potential 
Table 1 | Selected examples of modern genetically encoded indicators and their degree of utility in in vivo imaging in the mammalian brain.

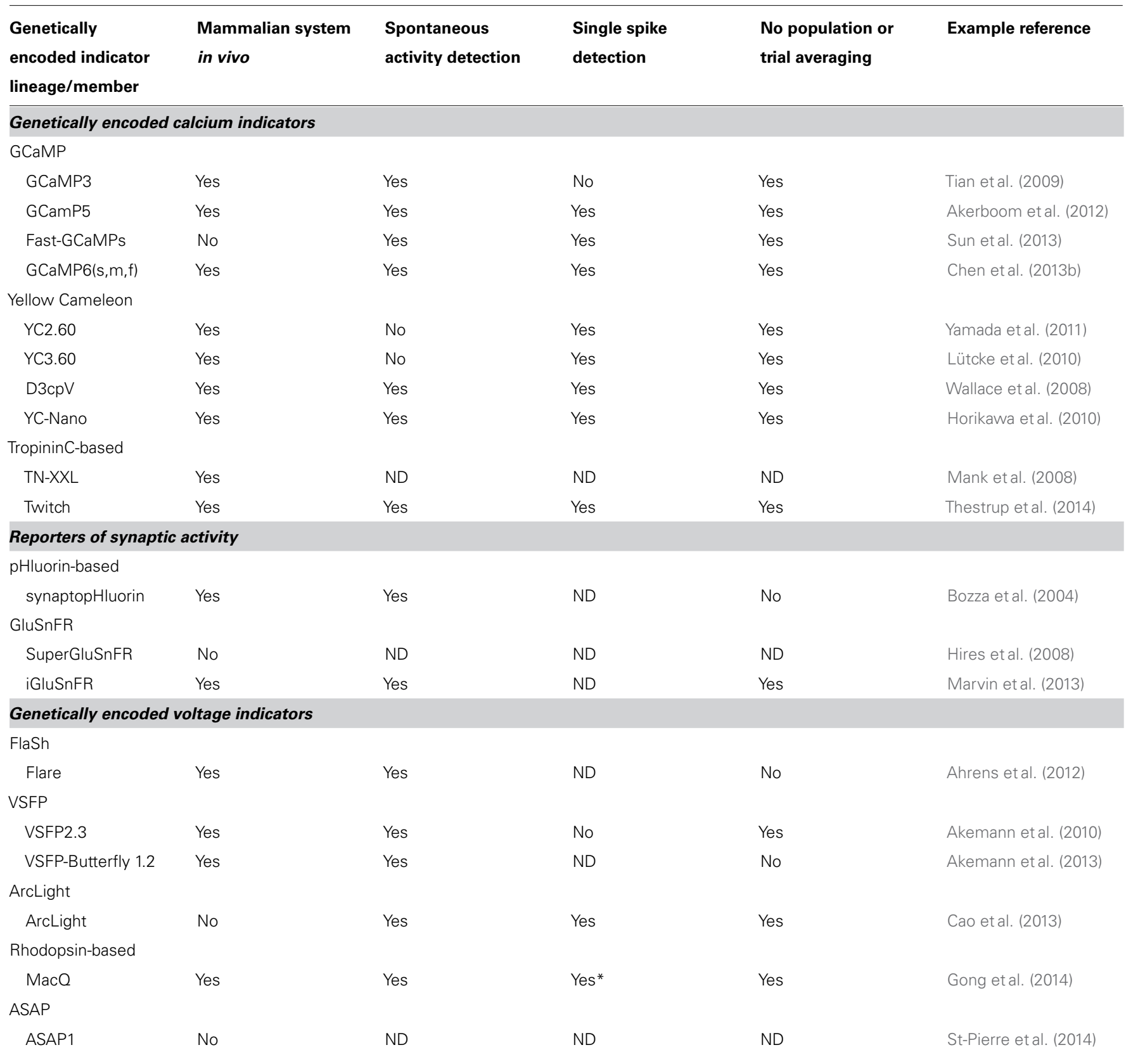

*Detected long-lasting $\mathrm{Ca}^{2}+$ spikes only. Shorter duration $\mathrm{Na}^{+}$spikes were not resolved.

ND, not determined.

of GECIs in multiplex imaging. Multi-color imaging allows simultaneous assay of diverse cell types. For example, different color probes will aid in the elucidation of the interplay between neurons and astrocytes in shaping the neural circuitry. Red-shifted indicators will additionally reduce tissue scattering (for both excitation and emission), phototoxicity, and background fluorescence, facilitating deep imaging. Finally, color-shifted indicators will seamlessly integrate into imaging experiments with other types of indicators and optogenetic tools (for review, see Akerboom et al., 2013).
For the past years, GECIs have driven the expansion of knowledge about the dynamics of neural circuitry gained through in vivo imaging of the intact brain (For details, see Recent Findings through GINA Technologies). GECI variants continue to open up new applications in neuroscience. Transgenic expression of inverse pericam and camgaroo-2 allowed the first detection of calcium signals evoked via sensory manipulation in a single trial in mouse (Hasan et al., 2004). D3cpv produced the first recordings of single spikes from mouse cortical cells in vivo (Wallace et al., 2008). Several GINAs have been deployed under a glial 
fibrillary acidic protein (GFAP) promoter in mice to allow readout of astrocytic activity in acute slice (Haustein et al., 2014) and in the intact, anesthetized animal (Atkin et al., 2009; Hoogland et al., 2009). Members of the TN-L (TN-XXL; Heider et al., 2010) and GCaMP (GCaMP5; Yin et al., 2014) lineages have recently been deployed for imaging studies in non-human primates. Future work within this model organism will produce results more pertinent to the human nervous system. GCaMP6 was used to report calcium transients from dendritic spines of excitatory and the dendrites of inhibitory interneurons located in primary visual cortex (Chen et al., 2013b). All of these milestones continue to demonstrate that GECIs have been a useful tool for breaking down barriers to advance our understanding of the dynamics of neural circuitry.

Despite the fact that calcium transients are an indirect measure of systems level circuit function, their superior SNR values relative to other GINA classes have assured their continued dominance in this arena for the near future. Though the advance of monitoring circuitry function depends on the improvement of genetically encoded voltage indicators (GEVIs) and other sensors probing all kinds of neural activity-for example transient changes to neuromodulator concentration in the extracellular space-GECIs will continue to find utility in the study of electrically silent cells which interact with brain circuitry, such as astrocytes.

\section{SENSORS REPORTING SYNAPTIC ACTIVITY}

Rapid information flow in the brain is mediated by anatomical connections at synapses between cells. During the course of an AP, membrane depolarization invades the presynaptic terminal. As noted above, this results in intracellular calcium transients, which in turn drive the fusion of neurotransmitter containing vesicles with the presynaptic cell membrane. This fusion event results in an immediate increase in the vesicular $\mathrm{pH}$, which is maintained at an acidic level, as the lumenal volume is expelled into the extracellular space. The contents of the vesicle are also released into the synaptic cleft, leading to a local increase in the concentration of neurotransmitter (Hille, 2001).

To better access synaptic transmission with optical tools, a variety of GINA classes have been developed. The first of these, synaptopHluorin, is derived from the fusion of a $\mathrm{pH}$ sensitive, ecliptic GFP variant at the c-terminus of the vesicular protein, synaptobrevin [also known as vesicular associated membrane protein-2 (VAMP2)], which localizes the FP to the vesicular lumen. The fluorescence of the ecliptic pHluorin is quenched by the acidic environment of synaptic vesicles ( $\mathrm{pH}$ 5.6). During neurotransmitter release, vesicles fuse with the plasma membrane, exposing the lumen to the neutral $\mathrm{pH}$ of the extracellular environment ( $\mathrm{pH} 7.4$ ), causing a dramatic increase in fluorescence intensity. The fluorescence intensity is then quenched once again after reconstitution and reacidification of the vesicle interior (Miesenbock et al., 1998; Figure 1D). SynaptopHluorin was one of the first GINAs to be employed for in vivo imaging in the mammalian system (Bozza et al., 2004).

Later modifications to these probes included fusions to different proteins which localize with higher specificity to synaptic vesicles such as synaptophysin (Granseth et al., 2006) and the vesicular glutamate transporter (VGLUT-1; Balaji and Ryan, 2007). Redshifted variants of synaptopHluorin have also been developed for multiplex imaging. These were constructed by exchanging the GFP based pHluorin with relatively red shifted FPs and include VGLUT1-mOrange2 (Li et al., 2011) and sypHTomato (Li and Tsien, 2012). Each of these probes was used concurrently with a GCaMP indicator to simultaneously probe presynaptic calcium signaling and presynaptic release at the same synapses or in distinct pre- and postsynaptic elements. These studies serve as early examples of the future possibilities presented by multiplex imaging.

Genetically encoded indicators of neural activity for probing excitatory neurotransmitter glutamate release and spillover have also been developed. Fluorescent indicator protein for glutamate (FLIPE; Okumoto etal., 2005) and the SuperGluSnFR (Tsien, 2005; Hires et al., 2008) are FRET-based probes which employ the linear fusion of periplasmic glutamate binding protein of Escherichia coli, GltI, (also known as ybeJ) with enhanced cyan fluorescent protein (ECFP) and a yellow FP, Citrine or Venus (Figures 1E,F). These reporters provide a sensitive optical readout of glutamate concentration in vitro by FRET-dependent changes in the CFP/YFP emission ratio. An improved version of GluSnFR, SuperGluSnFR has since been developed. Through linker optimization (Hires et al., 2008), SuperGluSNFR permits efficient optical measurements of the time course of synaptic glutamate release, spillover, and reuptake in cultured hippocampal neurons with centisecond temporal and dendritic spine-sized spatial resolution. Most recently, a single-FP based glutamate sensor, iGluSnFR, was developed. iGluSnFR is based on the fusion of Gltl with circularly permuted enhanced green fluorescent protein (cpEGFP; Figure 1E). Due to the superior SNR of this probe, it allows the resolution of glutamate transients at the apical tuft dendrites of layer $\mathrm{V}$ neurons in the motor cortex of awake, behaving mice (Marvin et al., 2013).

The development of sensors probing synaptic activity greatly expanded the kinds of neural activity that can be accessed by optical tools. The output activity of a neuron is sometimes thought of as being the voltage transients propagated to synaptic termini during an AP. Calcium transients are currently the most experimentally accessible marker of this phenomenon. However, under some conditions, the relationship between these signals and synaptic release is non-linear (see, e.g., Singer and Diamond, 2006). In this respect, reporters of synaptic activity will sometimes be a better choice of sensor for applications in which vesicle/transmitter release is the primary concern. Furthermore, neurotransmitter sensors allow detection of the presence of extra-synaptic neurotransmitter, which can have important functional consequences when interacting with neuronal receptors expressed distal to synapses (Marvin et al., 2013) and on astrocytes (Haustein et al., 2014).

\section{GENETICALLY ENCODED VOLTAGE INDICATORS}

Membrane potential undergoes a rapid change during the course of an AP as well as subthreshold oscillations. These changes can be succeeded by consequent changes to intracellular calcium and evoked synaptic signaling events (Hille, 2001). As such, voltage is considered the primary signal of interest and the most direct 
way to monitor neural activity. Despite this fact, imaging voltage is inherently difficult, largely due to the nature of the voltage signal itself. The short duration of the voltage change during APs (1-5 ms) demands faster kinetics and higher sensitivity of fluorescent sensors to yield sufficient photon budget for imaging (Peterka et al., 2011; Looger and Griesbeck, 2012). Additionally, GEVIs are necessarily membrane bound or associated, which reduces imaging volume relative to cytosolic GINAs (Peterka et al., 2011). However, recent advances in the field of protein engineering hold great promise for the development of GEVIs with superior SNR.

As demonstrated in Figures 1G,I,J, most GEVIs function as reporter of membrane voltage by tethering one or two FPs to a voltage sensitive protein derived from natural sources. The first genetically encoded voltage indicators - such as FlaSh (Siegel and Isacoff, 1997), sodium channel protein-based activity reporting construct (SPARC; Ataka and Pieribone, 2002), and voltage sensitive fluorescent protein 1 (VSFP 1; Sakai et al., 2001)-were based on intact voltage-gated potassium channels or their "voltagesensing" domains. However, these probes exhibit low SNR, slow kinetics and localize poorly to the membrane of mammalian cells (Baker et al., 2007).

The next generation of GEVIs was based on the voltage sensitive domain of a phospatase (VSP) derived from the sea squirt, Ciona intestinalis (Ci-VSP; Murata et al., 2005; Dimitrov et al., 2007; Akemann et al., 2010; Jin et al., 2012; Figures 1G,H). These probes include both FRET-based (Akemann et al., 2010) and single FP-based family (Lundby et al., 2008; Perron et al., 2009), and boasted much improved brightness, kinetics and improved membrane targeting relative to the earlier generation. One of the most advanced probes of this class, VSFP2.3, permitted in vivo recording of neural activity in mouse somatosensory cortex, despite poor cellular resolution. Recently, an improved variant, VSFP butterfly 1.2 (Figure 1G), showed increased SNR and allowed two-photon imaging of membrane changes produced by layer $2 / 3$ neurons of mouse barrel cortex, which represents the first reported use of at depth cellular resolution imaging by a GEVI. However, extensive trial averaging was required in order to resolve the signal (Akemann et al., 2013).

In parallel, protein engineering efforts have also led to single-FP based GEVIs. For example, the ArcLight (Figure 1H) fluorescence voltage sensors consist of the fusion of ecliptic pHluorin GFP to Ci-VSP and show significantly improved sensitivity in response to APs compared to other Ci-VSP based probes (Cao et al., 2013). More recently, Accelerated Sensor of Action Potentials 1 (ASAP1) has been developed in which circularly permuted green fluorescent protein (cpGFP) is inserted in an extracellular loop of a voltagesensing domain of a VSP derived from a chicken (Gallus gallus). ASAP-1 showed faster kinetics compatible with the typical 2-ms duration of APs and is capable of probing high-frequency AP trains (St-Pierre et al., 2014), thus permitting detection of subthreshold potential and hyperpolarization waveforms in cultured hippocampal neurons. However, voltage imaging using both ArcLight and ASAP-1 in intact rodent brains remains to be demonstrated.

In addition, another recent class of GEVI including PROPS (Kralj et al., 2011) and VIP1 (Kralj et al., 2012), has been developed based on voltage-induced fluorescence modulation of the retinal cofactor of bacterial and archaeal rhodopsins. In these probes, the rhodopsin itself acts as the sensor domain while the retinal cofactor serves as the fluorescence reporter via direct fluorescence (Kralj et al., 2011, 2012; Gong et al., 2013) or acting as FRET acceptor (illustrated in Figure 1H; Gong et al., 2014). Relative to the Ci-VSP based sensors, this class of sensor is extremely fast with submillisecond kinetics, sufficient to resolve single AP or subthreshold membrane voltage fluctuations in cultured mammalian neurons. However, because these probes rely on a retinal cofactor as the sole fluorescence source, they exhibit extremely low brightness. The most recent FRET-opsin sensors offer fast kinetics (rise time $\sim 5 \mathrm{~ms}$ ) as well as higher brightness, which has allowed for reporting long-lasting complex spikes in the dendritic arbors of Purkinje cells in the cerebellum of a living mouse. These spikes exhibit slower dynamics than typical $\mathrm{Na}^{+}$spikes $(\sim 5-10 \mathrm{~ms})$, but nevertheless were apparently detected at sub-cellular resolution with single-trial precision (Gong et al., 2014), a first for this GINA class.

Though current state-of-the art GEVIs have been deployed for in vivo imaging, their broad use has remained limited in rodent brain as shown in Table 1. As incremental improvements are made to existing and future probes, GEVIs may finally facilitate systems-level, cellular-resolution voltage imaging in living behaving mammals.

Blogs discussing all GINA classes can be found at open websites such as Andrew Hires' Brain Windows ${ }^{1}$ and Guillame Dugué's OpenOptogenetics ${ }^{2}$.

\section{CASE STUDY: FROM GCaMP3 TO GCaMP6}

Extensive protein engineering efforts have improved the properties of GINAs, especially the GCaMP family, to the point that they now rival synthetic alternatives such as Oregon Green Bapta-1 in terms of kinetics and sensitivity. Here we review established sensor design and optimization pipelines (rational design combined with directed evolution) that have proven to be effective to improve the intrinsic properties of GCaMPs, leading to a family of high performing sensors, GCaMP3, GCaMP5, and GCaMP6(s,m,f; Figures 2A,B).

Based on GCaMP3 scaffold, an array of GCaMP5 variants was generated by combining improvements from site-directed mutagenesis at cpEGFP/CaM interface and targeted library screening at the two linkers between cpEGFP and M13/CaM (Akerboom et al., 2012). Twelve GCaMP5s were systematically characterized in cultured neurons, astrocytes, and in Caenorhabditis elegans, drosophila, zebrafish, and mouse under various conditions. SNR was improved by at least twofold to threefold; all GCaMP5s showed improved dynamic range compared with GCaMP3. Users can choose from different GCaMP5 variants for different applications. Amongst these, GCaMP5A, GCaMP5G, and GCaMP5K exhibit the highest levels of general utility.

Based on the GCaMP5 scaffold, computational design has guided further targeting of 16 amino acid positions on the interface between cpEGFP and CaM at 18 positions including the M13CaM interface (Chen et al., 2013b). Four hundred and forty seven

\footnotetext{
${ }^{1}$ https://brainwindows.wordpress.com/

${ }^{2}$ http://www.openoptogenetics.org/
} 

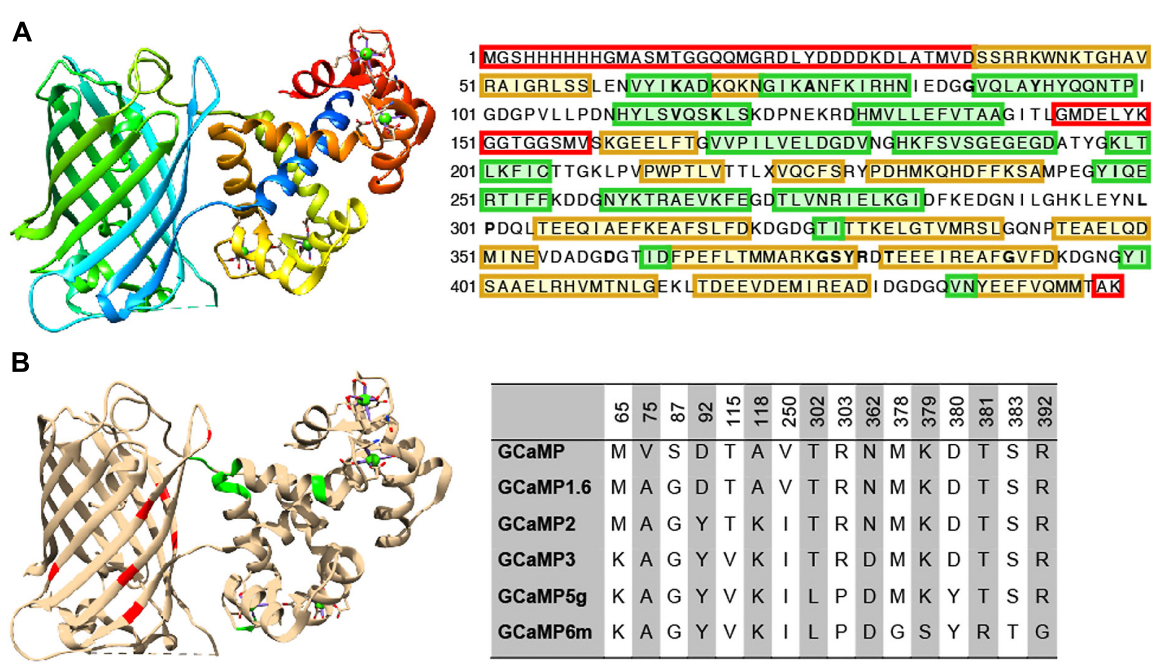

FIGURE 2 | Structure and beneficial mutations of GCaMPs. (A) Crystal structure (Ding etal., 2014) and primary sequence of GCaMP6m. Distinct regions of the crystal structure are indicated by color. The cooler the color, the closer the sequence is to $\mathrm{N}$-terminus. The primary sequence is annotated such that yellow shading corresponds to ß-strands, green shading corresponds to $\alpha$-helices, and residues in red are those that do not crystallize (i.e., pRSET domain and linkers). (B) Beneficial mutations of
GCaMPs. Crystal structure of GCaMP6m is annotated with iteratively evolved residues. Red residues are from the cpGFP and green ones are from calmodulin. Note that these residues are typically at the interface between the two domains. Residue matrix shows the evolution of GCaMP lineage with incrementally improved performance. Individual beneficial residues are highlighted in the primary sequence of GCaMP6m in (A).
GCaMP variants in were screened in dissociated neuronal culture resulting in three ultrasensitive GCaMP6 sensors with a variety of kinetics [i.e., GCaMP6s (slow), GCaMP6m (medium; Figure 2A), and GCaMP6f (fast)]. Compared to GCaMP5G, these sensors have 1.1- to 1.6-fold increase of dynamic range and threefold higher $\mathrm{Ca}^{2+}$ binding affinity. In addition, GCaMP6f shows the fastest kinetics among the entire GCaMP family due to a mutation at the M13-CaM interface (A317E).

Besides protein engineering efforts, the success of GCaMP6 development also depended on the establishment of a highly efficient high-throughput screening platform (Wardill et al., 2013). This system directly employed screening in dissociated rat cortical/hippocampal neuronal culture, bypassing the screening steps in bacterial and mammalian cells (Tian et al., 2009; Zhao et al., 2011). Specifically, lentivirus encoding GCaMP variants driven by human synapsin-1 promoter were produced to achieve dense and neuron-specific labeling in cultured neurons. To assist automated processing of imaging acquisition and analysis, internal ribosome entrance site (IRES) were introduced downstream of GCaMP to drive the expression of a second FP, nuclear targeted mCherry. The process of screening has been automated from probe development to imaging analysis (Wardill etal., 2013). This screening system can be easily adapted to aid in the optimization of other members of the GINA family.

As new members of the GCaMP family and other GINA classes are developed, their creators will often make them available for the larger research community. Frequently, genetic material encoding the probes can be acquired through Addgene ${ }^{3}$, a non-profit

\footnotetext{
${ }^{3}$ http://www.addgene.org
}

plasmid repository. For ready-made application, GINAs packaged into viral vectors can be obtained through viral core facilities of institutions such as the University of Pennsylvania ${ }^{4}$ and the University of North Carolina at Chapel Hill ${ }^{5}$. Finally, animals transgenic for several GINAs have been produced and are available for purchase through services such as those provided by the Jackson Laboratory ${ }^{6}$ and the Zebrafish International Resource Center ${ }^{7}$.

\section{IN VIVO IMAGING WITH GINAs IN LIVING, BEHAVING RODENT BRAIN}

Though some classes of GINAs have been optimized to the point that they are compatible for in vivo imaging, the noise level in vivo greatly challenges the SNR of the sensors, especially in the mammalian brain. To make imaging experiments meaningful and successful, consideration must be taken for proper selection of imaging configuration, expression systems, surgical procedure, imaging depth, and analytical techniques. To provide guidance for the end-users for choosing most suitable experimental settings, here we review a few important intrinsic and extrinsic parameters that are essential to maximize SNR and to achieve best imaging outcomes.

\section{GINA TRANSDUCTION METHODS}

The expression level of a GINA influences its SNR in cells and organisms in a complex manner. Low expression level will preclude

\footnotetext{
${ }^{4}$ http://www.med.upenn.edu/gtp/vectorcore/

${ }^{5} \mathrm{http}: / /$ www.med.unc.edu/genetherapy/vectorcore

${ }^{6}$ http://jaxmice.jax.org

${ }^{7}$ http://www.zebrafish.org
} 
sensor visualization and decrease SNR. It also demands imaging with higher laser power and longer exposure time, which may lead to phototoxicity, reduced imaging speed, and increased photobleaching. On the other hand, high expression level increases photon budget, but also increases substrate buffering (e.g., calcium by GECIs) and may perturb cell signaling pathways leading to cytotoxicity and cytomorbidity (see, e.g., Kuhn et al., 2012). Variation of expression level from cell to cell also impacts sensor performance, potentially confounding quantitative imaging analysis. To balance all these effects, it is essential to explore multiple promoters and regulatory sequences combined with the most suitable gene delivery methods to maximize the SNR of the sensor. Several methods of gene delivery have been optimized for the expression and subsequent recording of GINAs in the intact mammalian brain. These include introduction via stereotaxic viral injection, in utero electroporation, and stable transgenesis.

In the case of viral gene delivery, virus-encoding GINAs are injected directly into the brain region of interest (Wallace et al., 2008). Typically, recombinant adeno-associated virus (rAAV) has been broadly used due to its ability to diffuse easily away from the site of injection and relative simplicity (Grieger and Samulski, 2005). But others, such as adenoviral vector (e.g., Mohamed et al., 2013) and lentiviral vectors (e.g., Akerboom et al., 2012), are also in use (Packer et al., 2013). Combined with tissue specific promoters, such as synapsin-1, viral injection permits labeling of genetically defined local populations of neurons. The efficiency of probe expression can vary as a function of cell type, promoters, brain regions, and AAV serotype (Cearley and Wolfe, 2006; Wallace etal., 2008; Kuhn etal., 2012; Aschauer etal., 2013). For example, rAAV2 drives differential probe expression in cerebellar interneurons (Kuhn et al., 2012). However, when combined with specific promoter system, rAAV can be targeted to Purkinje cells in cerebellum cortex (Kuhn et al., 2012). In addition, rAAV9 is well suited for the transduction of cortical neurons (Cearley and Wolfe, 2006), while rAAV8 is particularly efficient in labeling astrocytes (Aschauer et al., 2013). Hybrid viruses have been developed which increase potential combinations of transduction efficiency and tropism. For example, AAV2/1 encoding GINAs have been widely used because they display a broad tropism within the central nervous system combined with high transduction efficiencies (Burger et al., 2004). Viral labeling results in long-term, relatively stable probe expression over months, during which time imaging experiments can be performed repeatedly (Mank et al., 2008; Tian et al., 2009; Kuhn etal., 2012; Margolis et al., 2012). The popularity of this method of gene transfer is due in large part to its relatively easy adoption with flexibility in choosing tool payload and injection locations.

In utero electroporation is a process in which plasmid is injected, usually to the ventricles of an animal during the prenatal period of its life. The plasmid is driven into cells at the ventricular surface via application of electrical current across the target structure. Due to the temporal pattern of laminar development and spatial pattern of cell lineages present in the cortex, the time and location at which the procedure is performed can restrict probe expression to specific layers (Tabata and Nakajima, 2001) or cell populations (Borrell et al., 2005) within the cortex. However, the expression level of GINAs driven by this method has been reported to cause cytotoxicity as expression begins during a developmentally sensitive period (Tian et al., 2009). Furthermore, the variation of expression level in cells appears more pronounced than that using viral transduction. Still, studies that are based around developmental questions of circuit formation can benefit from the use of this method.

Transgenesis refers to the permanent modification of the genome of an organism by the use of, for example, bacterial artificial chromosomes (Van Keuren et al., 2009), zinc finger nucleases (Urnov et al., 2010), transcription activator-like effector nucleases (Joung and Sander, 2013), or clustered regularly interspaced short palindromic repeats (CRISPR) associated genes (Mali et al., 2013). Transgenic rodent lines that stably express GINAs through multiple generations have been a standard tool in neuroscience. Transgenic lines have been engineered expressing GINAs such as inverse pericam and camgaroo-2 (Hasan et al., 2004), synaptopHluorin (Bozza et al., 2004), GCaMP2 (DíezGarcía et al., 2005), YC3.60 (Atkin et al., 2009), the hybrid voltage sensor (hVOS; Wang et al., 2012), and GCaMP3 under control of the Thy1 promoter (Zariwala et al., 2012). Gene transfer through transgenesis results in the stable and ubiquitous expression of the genetic construct in target cells throughout the organism, although expression levels tend to vary across lines and tissue regions (Zeng and Madisen, 2012). Studies that require dense sampling from a genetically defined population over the life course of an organism benefit from the use of transgenic organisms.

Targeting GINAs with adequate cell type specificity can be achieved through recombinase systems such as the Cre/loxP and FLP/FRT, especially by pairing recombinase-dependent viruses with recombinase-expressing animal lines. Selective expression of GINAs in subsets of anatomically or functionally related populations is possible by placing the GINA construct into the double-floxed inverted orientation (DIO) configuration (Atasoy et al., 2008) or using recently developed INTRSECT (Intronic recombinase sites enabling combinatorial targeting) strategy in which expression is contingent upon the presence of Boolean subsets of transcription factors (Fenno et al., 2014).

Pharmacological control can be exerted to achieve temporal control of GINA expression. For example, the Tet-On(Off) system causes expression(repression) of GINA expression in the presence of tetracycline or one of its analog (Gossen and Bujard, 1992; Hasan et al., 2004). Such temporal control can be important to prevent perturbation of early developmental processes or animal behavior, which may confound imaging results (Tian et al., 2009).

\section{OPTICAL INSTRUMENTATION}

One of the most vital components for successful in vivo fluorescence imaging in the mammalian brain is the microscopic system. Brain tissue is highly scattering to light and contains fluorophores, for example flavoproteins, with spectra which can overlap to a high degree with those of current GINAs. At the same time, the neural activity which is tracked by GINAs unfolds on the order of $1 \mathrm{~s}$ to 100 s of milliseconds, limiting the time-window for photon capture. Fluorescence microscopy including systems based on 
one photon (1P) or multiple photons have been developed and optimized to facilitate imaging experiments.

In one photon fluorescence microscopy, a fluorophore is initially bombarded with photons from a relatively high energy excitation light source. After absorption, some of the energy of the photon is lost through non-radiative processes. The fluorophore then emits lower energy photons which can be detected. Visible light employed in 1P microscopy scatters more easily in tissue because mean path length of a photon increases shorter wavelength. This effect limits the depth at which $1 \mathrm{P}$ systems are capable of imaging unaided to $\sim 100 \mu \mathrm{m}$. However, chargecoupled device and complementary metal oxide semiconductor detectors which can be used in conjunction with this imaging modality are capable of extremely fast $(>\mathrm{kHz})$ full field image acquisition rates. They are also typically relatively inexpensive when compared with comparable multi-photon alternatives (Helmchen and Denk, 2005).

In addition, 1P imaging light paths can be channeled through endoscopes (Flusberg et al., 2008; Barretto et al., 2011) as well as single and multimode fiber optic cable (Ferezou et al., 2006; Flusberg et al., 2008; Lütcke et al., 2010; Gunaydin et al., 2014) to image cells at depth within brain tissue. These systems can be deployed to image the brains of head-immobilized animals (Akemann et al., 2010) or miniaturized and mounted to a freely moving animal (Ghosh et al., 2011). As a note of caution, the introduction of an endoscope to brain tissue is highly invasive, while fiber-optic techniques generally result in data nearly devoid of fine-scale spatial information (although see, e.g., Ferezou et al., 2006), in which a braided fiber optic cable allowed the acquisition of coarse-grained spatial data).

Multi-photon-and in particular $2 \mathrm{P}$-microscopy has been the workhorse for in vivo imaging in the field of systems neuroscience (Denk etal., 1990). This is due primarily to two attractive, intrinsic features of these systems to reduce out of focus emission, light scattering, and phototoxicity. First, in multi-photon microscopy photon absorption varies non-linearly with excitation photon density. For example, this relationship is quadratic in the case of $2 \mathrm{P}$ microscopy. As a result, only fluorophores within the focal volume of the excitation light cone are activated. Second, the energy of multiple photons must combine to excite the fluorophore, meaning that lower energy (and thus, less scattering) excitation photons may be used (Denk et al., 1990).

Traditional 2P systems are limited in acquisition speed by the galvanometer mirrors, which give rise to low frame rate (up to $15 \mathrm{~Hz}$ at $512 \times 512$ frame size), but full flexibility of arbitrary scan geometries within a two-dimensional plane. Such geometric patterns can be extended into three dimensions by controlling axial motion with a piezoelectric focusing element, allowing fast, single line scans within this space (Göbel et al., 2007). Alternatively, resonant laser scan mirrors enable very fast scanning of high resolution full field frames $(512 \times 512$ pixels $)$ at 30 fps or higher with $8 \mathrm{kHz}$ scanners (Denk et al., 1990; Helmchen and Denk, 2005; Kerr and Denk, 2008). However, arbitrary scan patterns cannot be accommodated with a resonant mirror.

Imaging depths of $\sim 500 \mu \mathrm{m}$ using GINAs have been achieved routinely in $2 \mathrm{P}$ imaging while maintaining excellent spatial resolution in intact mammalian preparations (Helmchen and Denk, 2005). This depth has been increased through the use of adaptive optics, regenerative amplifiers (Theer et al., 2003; Mittmann et al., 2011) and three photon microscopy (Ouzounov etal., 2014) to $\sim 1000 \mu \mathrm{m}$, though the imaging depth can be increased up to $\sim 1600 \mu \mathrm{m}$ using small molecule dyes (Kobat et al., 2011). Greater depths can be achieved by using a fiber optic light path in conjunction with a piezoelectric driven actuator for raster scanning through the fiber (Helmchen et al., 2001; Flusberg et al., 2005; Sawinski et al., 2009). The relatively slow speed of image acquisition can be addressed through random access scanning of small regions of the volume of a cell or field of cells using acousto-optic devices to control the excitation scan path (Grewe et al., 2010; Katona et al., 2012; Fernández-Alfonso et al., 2014). Alternatively, multiple excitation beams can be multiplexed for near- simultaneous imaging of different spatial patterns (Cheng et al., 2011).

Widefield illumination with multi-photon techniques can be achieved through the use of spatial light patterning techniques such as spatial light modulation (Nikolenko et al., 2008) and the generalized phase contrast method (Palima and Gluckstad, 2008) as well temporal focusing (Oron and Silberberg, 2005; Papagiakoumou etal., 2010). When employed in conjunction, such techniques allow simultaneous excitation of large, 3D volumes. The resultant emission patterns can then be interrogated through a typical widefield microscopic detection apparatus (Papagiakoumou et al., 2010).

As imaging technologies such as these become more developed and widespread, GINAs will become ever more useful. Microscopes which are mountable to an unrestrained animal will allow recordings from animals in more ethologically relevant states. Technologies that produce faster frame rates in $2 \mathrm{P}$ imaging will allow denser sampling of neurons within a single recording epoch with current GECIs. These technologies will further be indispensable for constructing accurate recordings of future GEVIs expressed in mammalian systems.

\section{ACQUISITION OF MICROSCOPIC IMAGES}

Due to its propensity to scatter light, the skull presents a significant barrier to imaging an intact brain. However, it also serves to dampen pulsations arising from the movement of blood and cerebrospinal fluid through cerebral tissue. As a result, simply removing the cranium above the imaged region is not a common practice in modern GINA imaging. Typically, the skull's impact on image quality is reduced by either thinning the skull above the imaged region(s) (Grutzendler et al., 2002) or by inserting a glass cover slip above a craniotomy (Holtmaat et al., 2009). Both methods stabilize cellular position within brain tissue while allowing high-quality optical access.

Motion artifacts can further complicate imaging acquisition in living animals. Multiple approaches have been devised to reduce motion artifacts, including mechanical changes to the imaged tissue, animal restraint, and online and post hoc computational processing for maintaining image stability.

For $2 \mathrm{P}$ imaging, head fixation techniques to maintain the animal's position have been used in monkeys (Wurtz, 1968), flies (Dahmen, 1980), and rodents (Komisaruk, 1970). Head 
and body restraint allow stable imaging while both stimulus and behavioral response parameters can be tightly controlled. This, in turn, eases the task of quantifying neural activity with respect to these variables (Akemann et al., 2010; Andermann et al., 2010; Huber et al., 2012; Petreanu et al., 2012). Head restraint can be used in conjunction with a spherical (Dombeck et al., 2007) or linear (Marvin etal., 2013) treadmill, which allows the addition of assays which require locomotion on the part of the animal. Animals restrained in this manner can then be presented with passive stimuli (Niell and Stryker, 2010) or virtual sensory environments in which they can exert control over the stimulus via movement of the treadmill (Dombeck et al., 2010).

The microscope objective itself can also be stabilized relative to the imaged specimen via closed-loop designs whereby movement of the animal results in registered movement of the relevant optics. These systems function by detecting and correcting for motion in the axial direction based on general movement of tissue (e.g., Laffray et al., 2011) or by modeling and avoiding (Paukert and Bergles, 2012) or counteracting (Chen et al., 2013a) movement due to specific motion artifacts relevant to the experimental settings.

In addition, a number of algorithms have been developed for motion correction. These algorithms function by appropriately reshaping images in a time series such that consecutive frames are aligned. Algorithms developed for this purpose are based on Hidden Markov Models (Dombeck et al., 2010), crosscorrelation based registration (Guizar-Sicairos et al., 2008), or gradient-descent based minimization of image differences (Greenberg and Kerr, 2009). Besides computational approaches, FRET sensors or single FP sensors expressed along with a reference FP can be used to cancel the effects of motion artifact.

\section{IMAGE PROCESSING}

Image processing is a necessary step, as signals must be assigned to specific cells or subcellular compartments and described according to their content. A large array of approaches for analyzing and visualizing GINA data has been developed. One of the first questions addressed using such methods was image segmentation (i.e., what parts of the imaged field contain responsive elements) and spike or event detection (i.e., when does the fluorescence contained within these elements change significantly from baseline). Such approaches reduce variability and bias introduced to analysis by methods such as hand drawing of regions of interest (ROIs). Early algorithms developed for spike detection were based on simple thresholding of the $\Delta F / F$ signal [i.e., $\left(F-F_{0}\right) / F_{0}$ where $\mathrm{F}$ is the moment by moment spatially averaged fluorescence data and $F_{0}$ is typically defined as a time-averaged, background subtracted baseline fluorescence value] where event detection was triggered by this signal passing above the threshold (e.g., Mao et al., 2001). Later efforts have employed linear deconvolution (Yaksi and Friedrich, 2006; Holekamp, 2008; Vogelstein et al., 2010) or subtraction (Seelig et al., 2010) to detect occurrence of spiking events. These algorithms perform well in systems in which probe response is linear to the input signal. Other methods have used pixelwise correlation in the fluorescence signal (Ozden et al., 2008), principle components analysis followed by independent components analysis (Mukamel et al., 2009), Monte Carlo spike detection (Vogelstein etal., 2009), and supervised learning algorithms (Valmianski et al., 2010).

As the size of imaging datasets grows ever larger, it becomes increasingly critical to extract salient features within and across populations of cells expressing GINAs, especially correlating the activity of cells to the behavioral output of an animal. Such approaches can correlate the activity of single cells to behavioral data by simple coincidence (Seelig et al., 2010), regression (Miri et al., 2011), or machine-learning based classification schemes (Huber etal., 2012; Petreanu et al., 2012). Additionally, dynamics of entire cell populations can be visualized in low dimensional space by methods based on factor analysis of the dataset (Harvey etal., 2012). Recently, many of the most successful approaches examined above have been integrated into a suite of open source tools optimized for the analysis of large datasets termed "THUNDER" (Freeman et al., 2014) which can be accessed at http://thefreemanlab.com/thunder/. Taken together, the computational approaches for imaging analysis and visualization represent an indispensable set of tools for interpreting the vast quantities of data acquired in the course of GINA-enabled experiments.

\section{RECENT FINDINGS THROUGH GINA TECHNOLOGIES}

Genetically encoded indicators of neural activity attributesparticularly genetic specificity and long-term expression-make them excellent tools for circuit interrogation at multiple spatial and temporal scales. They have thus been used in recent years to make fundamental contributions to our understanding of brain function. In addition, several GINA classes have made important strides toward becoming standard tools for various applications in the study of mammalian neural circuitry in vivo.

\section{TOPOGRAPHICAL REPRESENTATIONS}

Topographical maps are systematic variations in the spatial layout of the response properties of a brain region which reflects the organization of a stimulus space (Hubel and Wiesel, 1959; Felleman and Van Essen, 1991). While electrophysiological techniques have generally been used to develop mesoscopic-scale [order of $100 \mu \mathrm{m}$ (Knöpfel, 2012)] mappings of brain regions, the application of GINAs in topographic mapping provides single cell spatial resolution. In addition, GINAs make it straightforward to rapidly transition between spatial scales, acquiring data from the cellular or population level or anywhere in between by simply changing image magnification.

The activity of genetically specified elements of a neural circuit can be recorded using GINAs. Topographical mapping of odorant to region of the rodent olfactory bulb has been described using GINAs transgenic animals (e.g., Hasan et al., 2004) or by intrinsic optical signals. Indeed, both techniques report a systematic olfactory map of homologous aliphatic compounds of increasing length (Johnson et al., 1999; Meister and Bonhoeffer, 2001; Fletcher et al., 2009). Only imaging studies with GINA, however, were able to leveraged the genetic specificity of GINAs to discern that the aliphatic odorant map is formed de novo within the 
postsynaptic circuitry of the olfactory bulb (Bozza et al., 2004; Fletcher et al., 2009).

One of the most powerful aspects of GINAs in studying topographical maps is the ability to allow for imaging cellular activity at spatial scales ranging from subcellular to millimeter, providing researcher to inspect the proverbial forest, individual trees, or both, all while maintaining awareness of current location. For example, a conspicuous lack of correlation between CA1 place cell location and the space they encoded has been demonstrated through cellular resolution imaging of virally driven GCaMP3 (Dombeck et al., 2010). In another recent study, optical recording auditory cortex at different spatial scales has resolved coarse and then cellular scale cortical activity in response to different tones. These results support the existence of a tonotopic axis along primary auditory cortex whose strength had been called into questions by previous studies (Issa et al., 2014).

\section{FUNCTIONAL ORGANIZATION OF NEURAL CIRCUITRY}

Local circuits within different cortical regions receive inputs from multiple distant brain regions. These inputs impact ongoing computations within, and thus the output from, local circuitry. In terms of input, a critical question is: what information is conveyed from distal brain regions to the local area? Several recent studies employed GECIs to record calcium transients in distal boutons of, for example cortico-cortical (Petreanu et al., 2012) and medial septal nucleus-hippocampal axons (Kaifosh et al., 2013) to address this question. Such studies have allowed unprecedented access to communication from one brain region to another with both cellular and genetic resolution.

Genetically encoded calcium indicators have also been used in addressing whether response properties of local cells within a region are a function of inputs received from hierarchically lower brain regions, or if those properties arise from local circuit interactions. For example, Glickfeld et al. (2013) demonstrated that the response properties of boutons projected from primary visual cortex in mouse to higher visual areas matched the response properties of cells within each region. This finding stands in contrast to the results from Fletcher etal. (2009) who demonstrated a de novo map of olfactory space that arises through local circuit interaction. In each case, GINAs were useful in disambiguating the degree to which local circuit computations result in new response properties.

Taken together, these studies point to the ways in which GINAs aid decoding of communication between brain regions, especially, revealing what message is sent between regions and how that message influences processing within the target structure. As probes of multiple colors become available for in vivo use, it will become possible to address such questions within a single experiment.

\section{STABILITY AND PLASTICITY OF NEURAL CIRCUITRY}

Neural circuitry is constantly reorganized structurally and functionally in response to experience and other influences on sensory input. Much effort has been dedicated in recent decades to determining under what conditions neural responses to a stimulus remain stable over time (Gerrow and Triller, 2010).
Genetically encoded indicators of neural activity are particularly well suited to the study of how neural responses to stimuli change over time. Because these probes express at roughly constant levels for periods as long as months, identified cells from a single organism can be repeatedly imaged over a long period of time (Mank et al., 2008; Tian et al., 2009; Kuhn et al., 2012; Margolis et al., 2012). As an example, Mank et al. (2008) showed that layer $2 / 3$ cells in primary visual cortex of mice expressing TN-XXL exhibit stable tuning to orientation when imaged over the course of weeks. Another study, in contrast, found that at a cellular level, hippocampal place cell response to place fields is highly variable over a similar period of time. Despite the fact that most cells did not retain consistent mapping with respect to the place fields, at the population level an accurate spatial map was preserved (Ziv et al., 2013).

Genetically encoded calcium indicators has also been used to probe the dynamic changes of neural ensembles in response to learning. For example, it was found that as mice were trained to perform a sensorimotor task, preferred stimuli of individual L2/3 neurons expressing GCaMPs in vibrissal motor cortex shifted over time. At the population level, however, activity became increasingly stereotyped and time-locked to the stimulus and motor outputs (Huber et al., 2012). These results suggest that L2/3 neurons in mice motor cortex integrate sensory input to task-related motor programs.

Finally, sensory deprivation has been shown to result in a rapid rescaling of synaptic weights that act to preserve mean levels of neuronal activity around a homeostatically regulated set point via in vivo calcium imaging using GCaMPs.(Turrigiano et al., 1998; Bishop and Zito, 2013). In this study the activity of L2/3 and L5 neurons expressing GCAMP3/5 in primary visual cortex of mice was monitored. It was shown that bilateral retinal lesion resulted in a rapid decrease in population activity, followed by recovery to baseline activity levels over the course of the next $24 \mathrm{~h}$ (Bishop and Zito, 2013).

\section{FUTURE PERSPECTIVES}

In this review, we have presented a broad overview of the history, current use, and future prospects for the use of GINAs in imaging neural activity in the intact mammalian brain. Through iterative optimization, GINAs - and particularly GECIs - have come to represent an important tool set for systems neuroscientists.

Despite these gains, the power of GINAs in different experimental approaches remains to be fully realized. Of particular importance, optimizing GEVIs to the point that they are widely applicable for in vivo imaging is an active area of protein engineering research. Synaptic transmission is another rich area of research that can be better accessed optically through developing novel GINAs that permit probing neurotransmitters, neuropeptides and neuromodulators at the single neuron level as well as at circuit level. For all GINA classes, further development and optimization of color-shifted variants will enhance options for multiplexing optical genetic tools within a single experiment.

The lessons learned and disciplined methods used in optimizing GECIs can be easily adapted for optimizing properties of other GINA classes. As these goals are achieved in the coming years, 
expect further fundamental contributions to our understanding of brain function to be derived through the use of GINAs in awake, behaving mammals.

\section{REFERENCES}

Ahrens, K. F., Heider, B., Lee, H., Isacoff, E. Y., and Siegel, R. M. (2012). Two-photon scanning microscopy of in vivo sensory responses of cortical neurons genetically encoded with a fluorescent voltage sensor in rat. Front. Neural Circuits. 6:15. doi: 10.3389/fncir.2012.00015

Ai, H. W., Baird, M. A., Shen, Y., Davidson, M. W., and Campbell, R. E. (2014). Engineering and characterizing monomeric fluorescent proteins for live-cell imaging applications. Nat. Protoc. 9, 910-928. doi: 10.1038/nprot.2014.054

Akemann, W., Mutoh, H., Perron, A., Rossier, J., and Knöpfel, T. (2010). Imaging brain electric signals with genetically targeted voltage-sensitive fluorescent proteins. Nat. Methods 7, 643-649. doi: 10.1038/nmeth.1479

Akemann, W., Sasaki, M., Mutoh, H., Imamura, T., Honkura, N., and Knöpfel, T. (2013). Two-photon voltage imaging using a genetically encoded voltage indicator. Sci. Rep. 3, 2231. doi: 10.1038/srep02231

Akerboom, J., Carreras Calderón, N., Tian, L., Wabnig, S., Prigge, M., Tolö, J., et al. (2013). Genetically encoded calcium indicators for multi-color neural activity imaging and combination with optogenetics. Front. Mol. Neurosci. 6:2. doi: 10.3389/fnmol.2013.00002

Akerboom, J., Chen, T. W., Wardill, T. J., Tian, L., Marvin, J. S., Mutlu, S., et al. (2012). Optimization of a GCaMP calcium indicator for neural activity imaging. J. Neurosci. 32, 13819-13840. doi: 10.1523/JNEUROSCI.2601-12.2012

Andermann, M. L., Kerlin, A. M., and Reid, R. C. (2010). Chronic cellular imaging of mouse visual cortex during operant behavior and passive viewing. Front. Cell. Neurosci. 4:3. doi: 10.3389/fncel.2010.00003

Aramuni, G., and Griesbeck, O. (2013). Chronic calcium imaging in neuronal development and disease. Exp. Neurol. 242, 50-56. doi: 10.1016/j.expneurol.2012.02.008

Aschauer, D. F., Kreuz, S., and Rumpel, S. (2013). Analysis of transduction efficiency, tropism and axonal transport of AAV serotypes 1, 2, 5, 6, 8 and 9 in the mouse brain. PLoS ONE 8:e76310. doi: 10.1371/journal.pone.0076310

Ashley, C. C., and Ridgway, E. B. (1968). Ridgway, Simultaneous recording of membrane potential, calcium transient and tension in single muscle fibers. Nature 219, 1168-1169. doi: 10.1038/2191168a0

Ataka, K., and Pieribone, V. A. (2002). A genetically targetable fluorescent probe of channel gating with rapid kinetics. Biophys. J. 82, 509-516. doi: 10.1016/S00063495(02)75415-5

Atasoy, D., Aponte, Y., Su, H. H., and Sternson, S. M. (2008). A FLEX switch targets Channelrhodopsin-2 to multiple cell types for imaging and long-range circuit mapping. J. Neurosci. 28, 7025-7030. doi: 10.1523/JNEUROSCI.195408.2008

Atkin, S. D., Patel, S., Kocharyan, A., Holtzclaw, L. A., Weerth, S. H., Schram, V., et al. (2009). Transgenic mice expressing a cameleon fluorescent $\mathrm{Ca}^{2+}$ indicator in astrocytes and Schwann cells allow study of glial cell $\mathrm{Ca}^{2+}$ signals in situ and in vivo. J. Neurosci. Methods 181, 212-226. doi: 10.1016/j.jneumeth.2009.05.006

Baird, G. S., Zacharias, D. A., and Tsien, R. Y. (1999). Circular permutation and receptor insertion within green fluorescent proteins. Proc. Natl. Acad. Sci. U.S.A. 96, 11241-11246. doi: 10.1073/pnas.96.20.11241

Baker, B. J., Lee, H., Pieribone, V. A., Cohen, L. B., Isacoff, E. Y., Knopfel, T., et al. (2007). Three fluorescent protein voltage sensors exhibit low plasma membrane expression in mammalian cells. J. Neurosci. Methods 161, 32-38. doi: 10.1016/j.jneumeth.2006.10.005

Balaji, J., and Ryan, T. A. (2007). Single-vesicle imaging reveals that synaptic vesicle exocytosis and endocytosis are coupled by a single stochastic mode. Proc. Natl. Acad. Sci. U.S.A. 104, 20576-20581. doi: 10.1073/pnas.0707574105

Barretto, R. P., Ko, T. H., Jung, J. C., Wang, T. J., Capps, G., Waters, A. C., et al. (2011). Time-lapse imaging of disease progression in deep brain areas using fluorescence microendoscopy. Nat. Med. 17, 223-228. doi: 10.1038/nm.2292

Ben Achour, S., Pont-Lezica, L., Béchade, C., and Pascual, O. (2010). Is astrocyte calcium signaling relevant for synaptic plasticity? Neuron Glia Biol. 6, 147-155. doi: 10.1017/S1740925X10000207

Bishop, H. I., and Zito, K. (2013). The downs and ups of sensory deprivation: evidence for firing rate homeostasis in vivo. Neuron 80, 247-249. doi: 10.1016/j.neuron.2013.10.011
Borrell, V., Yoshimura, Y., and Callaway, E. M. (2005). Targeted gene delivery to telencephalic inhibitory neurons by directional in utero electroporation. J. Neurosci. Methods 143, 151-158. doi: 10.1016/j.jneumeth.2004.09.027

Boulin, T., and Hobert, O. (2012). From genes to function: the C. elegans genetic toolbox. Wiley Interdiscip. Rev. Dev. Biol. 1, 114-137. doi: 10.1002/wdev.1

Bovetti, S., Moretti, C., and Fellin, T. (2014). Mapping brain circuit function in vivo using two-photon fluorescence microscopy. Microsc. Res. Tech. 77, 492-501. doi: 10.1002/jemt.22342

Bozza, T., McGann, J. P., Mombaerts, P., and Wachowiak, M. (2004). In vivo imaging of neuronal activity by targeted expression of a genetically encoded probe in the mouse. Neuron 42, 9-21. doi: 10.1016/S0896-6273(04)00144-8

Burger, C., Gorbatyuk, O. S., Velardo, M. J., Peden, C. S., Williams, P., Zolotukhin, S., et al. (2004). Recombinant AAV viral vectors pseudotyped with viral capsids from serotypes 1,2, and 5 display differential efficiency and cell tropism after delivery to different regions of the central nervous system. Mol. Ther. 10, 302-317. doi: 10.1016/j.ymthe.2004.05.024

Buzsaki, G. (2004). Large-scale recording of neuronal ensembles. Nat. Neurosci. 7, 446-451. doi: 10.1038/nn1233

Cao, G., Platisa, J., Pieribone, V. A., Raccuglia, D., Kunst, M., and Nitabach, M. N. (2013). Genetically targeted optical electrophysiology in intact neural circuits. Cell 154, 904-913. doi: 10.1016/j.cell.2013.07.027

Cearley, C. N., and Wolfe, J. H. (2006). Transduction characteristics of adenoassociated virus vectors expressing cap serotypes 7, 8, 9, and Rh10 in the mouse brain. Mol. Ther. 13, 528-537. doi: 10.1016/j.ymthe.2005.11.015

Chen, J. L., Pfäffli, O. A., Voigt, F. F., Margolis, D. J., and Helmchen, F. (2013a). Online correction of licking-induced brain motion during two-photon imaging with a tunable lens. J. Physiol. 591(Pt 19), 4689-4698. doi: 10.1113/jphysiol.2013. 259804

Chen, T. W., Wardill, T. J., Sun, Y., Pulver, S. R., Renninger, S. L., Baohan, A., et al. (2013b). Ultrasensitive fluorescent proteins for imaging neuronal activity. Nature 499, 295-300. doi: 10.1038/nature 12354

Cheng, A., Gonçalves, J. T., Golshani, P., Arisaka, K., and Portera-Cailliau, C. (2011). Simultaneous two-photon calcium imaging at different depths with spatiotemporal multiplexing. Nat. Methods 8, 139-142. doi: 10.1038/nme th. 1552

Crochet, S., Poulet, J. F., Kremer, Y., and Petersen, C. C. (2011). Synaptic mechanisms underlying sparse coding of active touch. Neuron 69, 1160-1175. doi: 10.1016/j.neuron.2011.02.022

Cui, G., Jun, S. B., Jin, X., Pham, M. D., Vogel, S. S., Lovinger, D. M., et al. (2013). Concurrent activation of striatal direct and indirect pathways during action initiation. Nature 494, 238-242. doi: 10.1038/nature11846

Dahmen, H. J. (1980). A simple apparatus to investigate the orientation of walking insects. Experientia 36, 685-687. doi: 10.1007/BF01970140

Denk, W., Strickler, J. H., and Webb, W. W. (1990). Two-photon laser scanning fluorescence microscopy. Science 248, 73-76. doi: 10.1126/science.2321027

Díez-García, J., Matsushita, S., Mutoh, H., Nakai, J., Ohkura, M., Yokoyama, J., et al. (2005). Activation of cerebellar parallel fibers monitored in transgenic mice expressing a fluorescent $\mathrm{Ca}^{2+}$ indicator protein. Eur. J. Neurosci. 22, 627-635. doi: 10.1111/j.1460-9568.2005.04250.x

Dimitrov, D., He, Y., Mutoh, H., Baker, B. J., Cohen, L., Akemann, W., etal. (2007). Engineering and characterization of an enhanced fluorescent protein voltage sensor. PLOS ONE 2:e440. doi: 10.1371/journal.pone. 0000440

Ding, J., Luo, A. F., Hu, L., Wang, D., and Shao, F. (2014). Structural basis of the ultrasensitive calcium indicator GCaMP6. Sci. China Life Sci. 57, 269-274. doi: 10.1007/s11427-013-4599-5

Ding, S. (2013). In vivo astrocytic $\mathrm{Ca}^{2+}$ signaling in health and brain disorders. Future Neurol. 8, 529-554. doi: 10.2217/fnl.13.38

Dombeck, D. A., Harvey, C. D., Tian, L., Looger, L. L., and Tank, D. W. (2010). Functional imaging of hippocampal place cells at cellular resolution during virtual navigation. Nat. Neurosci. 13, 1433-1440. doi: 10.1038/nn.2648

Dombeck, D. A., Khabbaz, A. N., Collman, F., Adelman, T. L., and Tank, D. W. (2007). Imaging large-scale neural activity with cellular resolution in awake, mobile mice. Neuron 56, 43-57. doi: 10.1016/j.neuron.2007.08.003

Felleman, D. J., and Van Essen, D. C. (1991). Distributed hierarchical processing in the primate cerebral cortex. Cereb. Cortex 1, 1-47. doi: 10.1093/cercor/1.1.1

Fenno, L. E., Mattis, J., Ramakrishnan, C., Hyun, M., Lee, S. Y., He, M., et al. (2014). Targeting cells with single vectors using multiple-feature Boolean logic. Nat. Methods 11, 763-772. doi: 10.1038/nmeth.2996 
Ferezou, I., Bolea, S., and Petersen, C. C. (2006). Visualizing the cortical representation of whisker touch: voltage-sensitive dye imaging in freely moving mice. Neuron 50, 617-629. doi: 10.1016/j.neuron.2006.03.043

Fernández-Alfonso, T., Nadella, K. M., Iacaruso, M. F., Pichler, B., Roš, H., Kirkby, P. A., et al. (2014). Monitoring synaptic and neuronal activity in 3D with synthetic and genetic indicators using a compact acousto-optic lens two-photon microscope. J. Neurosci. Methods 222, 69-81. doi: 10.1016/j.jneumeth.2013. 10.021

Fletcher, M. L., Masurkar, A. V., Xing, J., Imamura, F., Xiong, W., Nagayama, S., et al. (2009). Optical imaging of postsynaptic odor representation in the glomerular layer of the mouse olfactory bulb. J. Neurophysiol. 102, 817-830. doi: 10.1152/jn.00020.2009

Flusberg, B. A., Cocker, E. D., Piyawattanametha, W., Jung, J. C., Cheung, E. L., and Schnitzer, M. J. (2005). Fiber-optic fluorescence imaging. Nat. Methods 2 , 941-950. doi: 10.1038/nmeth820

Flusberg, B. A., Nimmerjahn, A., Cocker, E. D., Mukamel, E. A., Barretto, R. P., Ko, T. H., et al. (2008). High-speed, miniaturized fluorescence microscopy in freely moving mice. Nat. Methods 5, 935-938. doi: 10.1038/nmeth.1256

Freeman, J., Vladimirov, N., Kawashima, T., Mu, Y., Sofroniew, N, J., Bennett, D. V., et al. (2014). Mapping brain activity at scale with cluster computing. Nat. Methods 11, 941-950. doi: 10.1038/nmeth.3041

Gerrow, K., and Triller, A. (2010). Synaptic stability and plasticity in a floating world. Curr. Opin. Neurobiol. 20, 631-639. doi: 10.1016/j.conb.2010.06.010

Ghosh, K. K., Burns, L. D., Cocker, E. D., Nimmerjahn, A., Ziv, Y., Gamal, A. E., et al. (2011). Miniaturized integration of a fluorescence microscope. Nat. Methods 8 , 871-878. doi: 10.1038/nmeth.1694

Glickfeld, L. L., Andermann, M. L., Bonin, V., and Reid, R. C. (2013). Corticocortical projections in mouse visual cortex are functionally target specific. Nat Neurosci. 16, 219-226. doi: 10.1038/nn.3300

Göbel, W., Kampa, B. M., and Helmchen, F. (2007). Imaging cellular network dynamics in three dimensions using fast 3D laser scanning. Nat. Methods 4, 73-79. doi: 10.1038/nmeth989

Gong, Y., Li, J. Z., and Schnitzer, M. J. (2013). Enhanced archaerhodopsin fluorescent protein voltage indicators. PLoS ONE 8:e66959. doi: 10.1371/journal.pone.0066959

Gong, Y., Wagner, M. J., Li, J. Z., and Schnitzer, M. J. (2014). Imaging neural spiking in brain tissue using FRET-opsin protein voltage sensors. Nat. Commun. 5, 3674 doi: $10.1038 /$ ncomms4674

Gossen, M., and Bujard, H. (1992). Tight control of gene expression in mammalian cells by tetracycline-responsive promoters. Proc. Natl. Acad. Sci. U.S.A. 89, 5547 5551. doi: 10.1073/pnas.89.12.5547

Granseth, B., Odermatt, B., Royle, S. J., and Lagnado, L. (2006). Clathrin-mediated endocytosis is the dominant mechanism of vesicle retrieval at hippocampal synapses. Neuron 51, 773-786. doi: 10.1016/j.neuron.2006.08.029

Greenberg, D. S., and Kerr, J. N. (2009). Automated correction of fast motion artifacts for two-photon imaging of awake animals. J. Neurosci. Methods 176 1-15. doi: 10.1016/j.jneumeth.2008.08.020

Grewe, B. F., Langer, D., Kasper, H., Kampa, B. M., and Helmchen, F. (2010) High-speed in vivo calcium imaging reveals neuronal network activity with nearmillisecond precision. Nat. Methods 7, 399-405. doi: 10.1038/nmeth.1453

Grieger, J., and Samulski, R. (2005). “Adeno-associated virus as a gene therapy vector: vector development, production and clinical applications," in Gene Therapy and Gene Delivery Systems, eds D. Schaffer and W. Zhou (Heidelberg: Springer), 119145 .

Grutzendler, J., Kasthuri, N., and Gan, W. B. (2002). Long-term dendritic spine stability in the adult cortex. Nature 420, 812-816. doi: 10.1038/ nature 01276

Guizar-Sicairos, M., Thurman, S. T., and Fienup, J. R. (2008). Efficient subpixel image registration algorithms. Opt. Lett. 33, 156-158. doi: 10.1364/OL.33.000156

Gunaydin, L. A., Grosenick, L., Finkelstein, J. C., Kauvar, I. V., Fenno, L. E., Adhikari, A., et al. (2014). Natural neural projection dynamics underlying social behavior. Cell 157, 1535-1551. doi: 10.1016/j.cell.2014.05.017

Harvey, C. D., Coen, P., and Tank, D. W. (2012). Choice-specific sequences in parietal cortex during a virtual-navigation decision task. Nature 484, 62-68. doi: 10.1038/nature10918

Hasan, M. T., Friedrich, R. W., Euler, T., Larkum, M. E., Giese, G., Both, M., et al. (2004). Functional fluorescent $\mathrm{Ca}^{2+}$ indicator proteins in transgenic mice under TET control. PLoS Biol. 2:e163. doi: 10.1371/journal.pbio.0020163
Haustein, M. D., Kracun, S., Lu, X. H., Shih, T., Jackson-Weaver, O., Tong, X., et al. (2014). Conditions and constraints for astrocyte calcium signaling in the hippocampal mossy fiber pathway. Neuron 82, 413-429. doi: 10.1016/j.neuron.2014.02.041

Heider, B., Nathanson, J. L., Isacoff, E. Y., Callaway, E. M., and Siegel, R. M. (2010). Two-photon imaging of calcium in virally transfected striate cortical neurons of behaving monkey. PLoS ONE 5:e13829. doi: 10.1371/journal.pone.0013829

Helmchen, F., and Denk, W. (2005). Deep tissue two-photon microscopy. Nat. Methods 2, 932-940. doi: 10.1038/nmeth818

Helmchen, F., Fee, M. S., Tank, D. W., and Denk, W. (2001). A miniature head-mounted two-photon microscope. High-resolution brain imaging in freely moving animals. Neuron 31, 903-912. doi: 10.1016/S0896-6273(01)00421-4

Helmchen, F., Imoto, K., and Sakmann, B. (1996). $\mathrm{Ca}^{2+}$ buffering and action potential-evoked $\mathrm{Ca}^{2+}$ signaling in dendrites of pyramidal neurons. Biophys. J. 70, 1069-1081. doi: 10.1016/S0006-3495(96)79653-4

Higashijima, S., Masino, M. A., Mandel, G., and Fetcho, J. R. (2003). Imaging neuronal activity during zebrafish behavior with a genetically encoded calcium indicator. J. Neurophysiol. 90, 3986-3997. doi: 10.1152/jn.00576.2003

Hille, B. (2001). Ion Channels of Excitable Membranes, 3rd Edn. Sunderland, MA: Sinauer Associates Inc.

Hires, S. A., Zhu, Y., and Tsien, R. Y. (2008). Optical measurement of synaptic glutamate spillover and reuptake by linker optimized glutamate-sensitive fluorescent reporters. Proc. Natl. Acad. Sci. U.S.A. 105, 4411-4416. doi: 10.1073/pnas.0712008105

Holekamp, T. F. (2008). A Novel High-Speed Sectioning Fluorescence Microscope Reveals Patterned Neural Responses to Pheromones, Ph.D. thesis Program in Neurosciences, Washington University, Washington.

Holtmaat, A., Bonhoeffer, T., Chow, D. K., Chuckowree, J., De Paola, V., Hofer, S. B., et al. (2009). Long-term, high-resolution imaging in the mouse neocortex through a chronic cranial window. Nat. Protoc. 4, 1128-1144. doi: 10.1038/nprot.2009.89

Hoogland, T. M., Kuhn, B., Göbel, W., Huang, W., Nakai, J., Helmchen, F., et al. (2009). Radially expanding transglial calcium waves in the intact cerebellum. Proc. Natl. Acad. Sci. U.S.A. 106, 3496-3501. doi: 10.1073/pnas.0809269106

Horikawa, K., Yamada, Y., Matsuda, T., Kobayashi, K., Hashimoto, M., Matsuura, T., et al. (2010). Spontaneous network activity visualized by ultrasensitive $\mathrm{Ca}^{2+}$ indicators, yellow Cameleon-Nano. Nat. Methods 7, 729-732. doi: 10.1038/nmeth. 1488

Hubel, D. H., and Wiesel, T. N. (1959). Receptive fields of single neurones in the cat's striate cortex. J. Physiol. 148, 574-591.

Huber, D., Gutnisky, D. A., Peron, S., O’Connor, D. H., Wiegert, J. S., Tian, L., et al. (2012). Multiple dynamic representations in the motor cortex during sensorimotor learning. Nature 484, 473-478. doi: 10.1038/nature11039

Ibraheem, A., and Campbell, R. E. (2010). Designs and applications of fluorescent protein-based biosensors. Curr. Opin. Chem. Biol. 14, 30-36. doi: 10.1016/j.cbpa.2009.09.033

Issa, J. B., Haeffele, B. D., Agarwal, A., Bergles, D. E., Young, E. D., and Yue, D. T. (2014). Multiscale optical $\mathrm{Ca}^{2+}$ imaging of tonal organization in mouse auditory cortex. Neuron 83, 944-959. doi: 10.1016/j.neuron.2014.07.009

Jin, L., Han, Z., Platisa, J., Wooltorton, J. R., Cohen, L. B., and Pieribone, V. A. (2012). Single action potentials and subthreshold electrical events imaged in neurons with a fluorescent protein voltage probe. Neuron 75, 779-785. doi: 10.1016/j.neuron.2012.06.040

Johnson, B. A., Woo, C. C., Hingco, E. E., Pham, K. L., and Leon, M. (1999). Multidimensional chemotopic responses to n-aliphatic acid odorants in the rat olfactory bulb. J. Comp. Neurol. 409, 529-548. doi: 10.1002/(SICI)10969861(19990712)409:4<529::AID-CNE2>3.0.CO;2-N

Joung, J. K., and Sander, J. D. (2013). TALENs: a widely applicable technology for targeted genome editing. Nat. Rev. Mol. Cell Biol. 14, 49-55. doi: $10.1038 / \mathrm{nrm} 3486$

Kaifosh, P., Lovett-Barron, M., Turi, G. F., Reardon, T. R., and Losonczy, A. (2013). Septo-hippocampal GABAergic signaling across multiple modalities in awake mice. Nat. Neurosci. 16, 1182-1184. doi: 10.1038/nn.3482

Katona, G., Szalay, G., Maák, P., Kaszás, A., Veress, M., Hillier, D., et al. (2012). Fast two-photon in vivo imaging with three-dimensional random-access scanning in large tissue volumes. Nat. Methods 9, 201-208. doi: 10.1038/nmeth.1851

Kerr, J. N., and Denk, W. (2008). Imaging in vivo: watching the brain in action. Nat. Rev. Neurosci. 9, 195-205. doi: 10.1038/nrn2338 
Kerr, R., Lev-Ram, V., Baird, G., Vincent, P., Tsien, R. Y., and Schafer, W. R. (2000). Optical imaging of calcium transients in neurons and pharyngeal muscle of $C$. elegans. Neuron 26, 583-594. doi: 10.1016/S0896-6273(00)81196-4

Knöpfel, T. (2012). Genetically encoded optical indicators for the analysis of neuronal circuits. Nat. Rev. Neurosci. 13, 687-700. doi: 10.1038/nrn3293

Kobat, D., Horton, N. G., and Xu, C. (2011). In vivo two-photon microscopy to 1.6$\mathrm{mm}$ depth in mouse cortex. J. Biomed. Opt. 16, 106014. doi: 10.1117/1.3646209

Koester, H. J., and Sakmann, B. (2000). Calcium dynamics associated with action potentials in single nerve terminals of pyramidal cells in layer $2 / 3$ of the young rat neocortex. J. Physiol. 529(Pt 3), 625-646. doi: 10.1111/j.1469-7793.2000.00625.x

Komisaruk, B. R. (1970). Synchrony between limbic system theta activity and rhythmical behavior in rats. J. Comp. Physiol. Psychol. 70, 482-492. doi: $10.1037 / \mathrm{h} 0028709$

Kralj, J. M., Douglass, A. D., Hochbaum, D. R., Maclaurin, D., and Cohen, A. E. (2012). Optical recording of action potentials in mammalian neurons using a microbial rhodopsin. Nat. Methods 9, 90-95. doi: 10.1038/nmeth.1782

Kralj, J. M., Hochbaum, D. R., Douglass, A. D., and Cohen, A. E. (2011). Electrical spiking in Escherichia coli probed with a fluorescent voltage-indicating protein. Science 333, 345-348. doi: 10.1126/science.1204763

Kuhn, B., Ozden, I., Lampi, Y., Hasan, M. T., and Wang, S. S. (2012). An amplified promoter system for targeted expression of calcium indicator proteins in the cerebellar cortex. Front. Neural Circuits 6:49. doi: 10.3389/fncir.2012.00049

Laffray, S., Pagès, S., Dufour, H., De Koninck, P., De Koninck, Y., and Côté, D. (2011). Adaptive movement compensation for in vivo imaging of fast cellular dynamics within a moving tissue. PLoS ONE 6:e19928. doi: 10.1371/journal.pone.0019928

Li, H., Foss, S. M., Dobryy, Y. L., Park, C. K., Hires, S. A., Shaner, N. C., et al. (2011). Concurrent imaging of synaptic vesicle recycling and calcium dynamics. Front. Mol. Neurosci. 4:34. doi: 10.3389/fnmol.2011.00034

Li, Y., and Tsien, R. W. (2012). pHTomato, a red, genetically encoded indicator that enables multiplex interrogation of synaptic activity. Nat. Neurosci. 15, 1047-1053. doi: $10.1038 / \mathrm{nn} .3126$

Liu, B. H., Li, P., Li, Y. T., Sun, Y. J., Yanagawa, Y., Obata, K., et al. (2009). Visual receptive field structure of cortical inhibitory neurons revealed by two-photon imaging guided recording. J. Neurosci. 29, 10520-10532. doi: 10.1523/JNEUROSCI.1915-09.2009

Looger, L. L., and Griesbeck, O. (2012). Genetically encoded neural activity indicators. Curr. Opin. Neurobiol. 22, 18-23. doi: 10.1016/j.conb.2011.10.024

Lundby, A., Mutoh, H., Dimitrov, D., Akemann, W., and Knöpfel, T. (2008). Engineering of a genetically encodable fluorescent voltage sensor exploiting fast Ci-VSP voltage-sensing movements. PLoS ONE 3:e2514. doi: 10.1371/journal.pone.0002514

Lütcke, H., Murayama, M., Hahn, T., Margolis, D. J., Astori, S., Zum Alten Borgloh, S. M., et al. (2010). Optical recording of neuronal activity with a geneticallyencoded calcium indicator in anesthetized and freely moving mice. Front. Neural Circuits 4:9. doi: 10.3389/fncir.2010.00009

Mali, P., Esvelt, K. M., and Church, G. M. (2013). Cas9 as a versatile tool for engineering biology. Nat. Methods 10, 957-963. doi: 10.1038/nmeth.2649

Mank, M., Reiff, D. F., Heim, N., Friedrich, M. W., Borst, A., and Griesbeck, O. (2006). A FRET-based calcium biosensor with fast signal kinetics and high fluorescence change. Biophys. J. 90, 1790-1796. doi: 10.1529/biophysj.105. 073536

Mank, M., Santos, A. F., Direnberger, S., Mrsic-Flogel, T. D., Hofer, S. B., Stein, V., et al. (2008). A genetically encoded calcium indicator for chronic in vivo two-photon imaging. Nat. Methods 5, 805-811. doi: 10.1038/nmeth.1243

Mante, V., Sussillo, D., Shenoy, K. V., and Newsome, W. T. (2013). Contextdependent computation by recurrent dynamics in prefrontal cortex. Nature 503, 78-84. doi: 10.1038/nature12742

Mao, B. Q., Hamzei-Sichani, F., Aronov, D., Froemke, R. C., and Yuste, R. (2001). Dynamics of spontaneous activity in neocortical slices. Neuron 32, 883-898. doi: 10.1016/S0896-6273(01)00518-9

Margolis, D. J., Lütcke, H., Schulz, K., Haiss, F., Weber, B., Kügler, S., et al. (2012). Reorganization of cortical population activity imaged throughout long-term sensory deprivation. Nat. Neurosci. 15, 1539-1546. doi: 10.1038/nn.3240

Marvin, J. S., Borghuis, B. G., Tian, L., Cichon, J., Harnett, M. T., Akerboom, J., et al. (2013). An optimized fluorescent probe for visualizing glutamate neurotransmission. Nat. Methods 10, 162-170. doi: 10.1038/nmeth.2333

Meister, M., and Bonhoeffer, T. (2001). Tuning and topography in an odor map on the rat olfactory bulb. J. Neurosci. 21, 1351-1360.
Miesenbock, G., De Angelis, D. A., and Rothman, J. E. (1998). Visualizing secretion and synaptic transmission with $\mathrm{pH}$-sensitive green fluorescent proteins. Nature 394, 192-195. doi: 10.1038/28190

Miri, A., Daie, K., Arrenberg, A. B., Baier, H., Aksay, E., and Tank, D. W. (2011). Spatial gradients and multidimensional dynamics in a neural integrator circuit. Nat. Neurosci. 14, 1150-1159. doi: 10.1038/nn.2888

Mittmann, W., Wallace, D. J., Czubayko, U., Herb, J. T., Schaefer, A. T., Looger, L. L., et al. (2011). Two-photon calcium imaging of evoked activity from L5 somatosensory neurons in vivo. Nat. Neurosci. 14, 1089-1093. doi: 10.1038/nn.2879

Miyawaki, A. (2011). Development of probes for cellular functions using fluorescent proteins and fluorescence resonance energy transfer. Annu. Rev. Biochem. 80, 357-373. doi: 10.1146/annurev-biochem-072909-094736

Miyawaki, A., Griesbeck, O., Heim, R., and Tsien, R. Y. (1999). Dynamic and quantitative $\mathrm{Ca}^{2+}$ measurements using improved cameleons. Proc. Natl. Acad. Sci. U.S.A. 96, 2135-2140. doi: 10.1073/pnas.96.5.2135

Miyawaki, A., Llopis, J., Heim, R., McCaffery, J. M., Adams, J. A., Ikura, M., et al. (1997). Fluorescent indicators for $\mathrm{Ca}^{2+}$ based on green fluorescent proteins and calmodulin. Nature 388, 882-887. doi: 10.1038/42264

Mohamed, T. M., Abou-Leisa, R., Baudoin, F., Stafford, N., Neyses, L., Cartwright, E. J., et al. (2013). Development and characterization of a novel fluorescent indicator protein PMCA4-GCaMP2 in cardiomyocytes. J. Mol. Cell. Cardiol. 63, 57-68. doi: 10.1016/j.yjmcc.2013.07.007

Mukamel, E. A., Nimmerjahn, A., and Schnitzer, M. J. (2009). Automated analysis of cellular signals from large-scale calcium imaging data. Neuron 63, 747-760. doi: 10.1016/j.neuron.2009.08.009

Murata, Y., Iwasaki, H., Sasaki, M., Inaba, K., and Okamura, Y. (2005). Phosphoinositide phosphatase activity coupled to an intrinsic voltage sensor. Nature 435, 1239-1243. doi: 10.1038/nature03650

Muto, A., Ohkura, M., Abe, G., Nakai, J., and Kawakami, K. (2013). Real-time visualization of neuronal activity during perception. Curr. Biol. 23, 307-311. doi: 10.1016/j.cub.2012.12.040

Muto, A., Ohkura, M., Kotani, T., Higashijima, S., Nakai, J., and Kawakami, K. (2011). Genetic visualization with an improved GCaMP calcium indicator reveals spatiotemporal activation of the spinal motor neurons in zebrafish. Proc. Natl. Acad. Sci. U.S.A. 108, 5425-5430. doi: 10.1073/pnas.1000887108

Nagai, T., Sawano, A., Park, E. S., and Miyawaki, A. (2001). Circularly permuted green fluorescent proteins engineered to sense $\mathrm{Ca}^{2+}$. Proc. Natl. Acad. Sci. U.S.A. 98, 3197-3202. doi: 10.1073/pnas.051636098

Nagai, T., Yamada, S., Tominaga, T., Ichikawa, M., and Miyawaki, A. (2004). Expanded dynamic range of fluorescent indicators for $\mathrm{Ca}^{2+}$ by circularly permuted yellow fluorescent proteins. Proc. Natl. Acad. Sci. U.S.A. 101, 10554-10559. doi: 10.1073/pnas.0400417101

Nakai, J., Ohkura, M., and Imoto, K. (2001). A high signal-to-noise $\mathrm{Ca}^{2+}$ probe composed of a single green fluorescent protein. Nat. Biotechnol. 19, 137-141. doi: $10.1038 / 84397$

Niell, C. M., and Stryker, M. P. (2010). Modulation of visual responses by behavioral state in mouse visual cortex. Neuron 65, 472-479. doi: 10.1016/j.neuron.2010.01.033

Nikolenko, V., Watson, B. O., Araya, R., Woodruff, A., Peterka, D. S., and Yuste, R. (2008). SLM microscopy: scanless two-photon imaging and photostimulation with spatial light modulators. Front. Neural Circuits 2:5. doi: 10.3389/neuro.04.005.2008

Ohkura, M., Matsuzaki, M., Kasai, H., Imoto, K., and Nakai, J. (2005). Genetically encoded bright $\mathrm{Ca}^{2+}$ probe applicable for dynamic $\mathrm{Ca}^{2+}$ imaging of dendritic spines. Anal. Chem. 77, 5861-5869. doi: 10.1021/ ac0506837

Ohkura, M., Sasaki, T., Sadakari, J., Gengyo-Ando, K., Kagawa-Nagamura, Y., Kobayashi, C., et al. (2012). Genetically encoded green fluorescent $\mathrm{Ca}^{2+}$ indicators with improved detectability for neuronal $\mathrm{Ca}^{2+}$ Signals. PLoS ONE 7:e51286. doi: 10.1371/journal.pone.0051286

Okumoto, S., Looger, L. L., Micheva, K. D., Reimer, R. J., Smith, S. J., and Frommer, W. B. (2005). Detection of glutamate release from neurons by genetically encoded surface-displayed FRET nanosensors. Proc. Natl. Acad. Sci. U.S.A. 102, 87408745. doi: 10.1073/pnas.0503274102

Oron, D., and Silberberg, Y. (2005). Spatiotemporal coherent control using shaped, temporally focused pulses. Opt. Express 13, 9903-9908. doi: 10.1364/OPEX.13.009903 
Ouzounov, D. G., Horton, N., Wang, T., Feng, D., Nishimura, N., and Xu, C. (2014). "In vivo three-photon calcium imaging of brain activity from layer 6 neurons in mouse brain," in CLEO, Postdeadline Paper Digest (San Jose, CA: Optical Society of America). doi: 10.1364/CLEO_SI.2014.STh5C.2

Ozden, I., Lee, H. M., Sullivan, M. R., and Wang, S. S. (2008). Identification and clustering of event patterns from in vivo multiphoton optical recordings of neuronal ensembles. J. Neurophysiol. 100, 495-503. doi: 10.1152/jn.01310.2007

Packer, A. M., Roska, B., and Hausser, M. (2013). Targeting neurons and photons for optogenetics. Nat. Neurosci. 16, 805-815. doi: 10.1038/nn.3427

Palima, D., and Gluckstad, J. (2008). Comparison of generalized phase contrast and computer generated holography for laser image projection. Opt. Express 16, 5338-5349. doi: 10.1364/OE.16.005338

Palmer, A. E., Giacomello, M., Kortemme, T., Hires, S. A., Lev-Ram, V., Baker, D. et al. (2006). $\mathrm{Ca}^{2+}$ indicators based on computationally redesigned calmodulinpeptide pairs. Chem. Biol. 13, 521-530. doi: 10.1016/j.chembiol.2006.03.007

Palmer, A. E., Qin, Y., Park, J. G., and McCombs, J. E. (2011). Design and application of genetically encoded biosensors. Trends Biotechnol. 29, 144-152. doi: 10.1016/j.tibtech.2010.12.004

Papagiakoumou, E., Anselmi, F., Bègue, A., de Sars, V., Glückstad, J., Isacoff, E. Y., et al. (2010). Scanless two-photon excitation of channelrhodopsin-2. Nat. Methods 7, 848-854. doi: 10.1038/nmeth.1505

Paukert, M., and Bergles, D. E. (2012). Reduction of motion artifacts during in vivo two-photon imaging of brain through heartbeat triggered scanning. J. Physiol. 590(Pt 13), 2955-2963. doi: 10.1113/jphysiol.2012.228114

Perea, G., and Araque, A. (2005). Glial calcium signaling and neuron-glia communication. Cell Calcium 38, 375-382. doi: 10.1016/j.ceca.2005.06.015

Perron, A., Mutoh, H., Launey, T., and Knöpfel, T. (2009). Red-shifted voltage-sensitive fluorescent proteins. Chem. Biol. 16, 1268-1277. doi: 10.1016/j.chembiol.2009.11.014

Peterka, D. S., Takahashi, H., and Yuste, R. (2011). Imaging voltage in neurons. Neuron 69, 9-21. doi: 10.1016/j.neuron.2010.12.010

Petreanu, L., Gutnisky, D. A., Huber, D., Xu, N. L., O’Connor, D. H., Tian, L., et al. (2012). Activity in motor-sensory projections reveals distributed coding in somatosensation. Nature 489, 299-303. doi: 10.1038/nature11321

Polikov, V. S., Tresco, P. A., and Reichert, W. M. (2005). Response of brain tissue to chronically implanted neural electrodes. J. Neurosci. Methods 148, 1-18. doi: 10.1016/j.jneumeth.2005.08.015

Reid, R. C., Victor, J. D., and Shapley, R. M. (1997). The use of m-sequences in the analysis of visual neurons: linear receptive field properties. Vis. Neurosci. 14, 1015-1027. doi: 10.1017/S0952523800011743

Romoser, V. A., Hinkle, P. M., and Persechini, A. (1997). Detection in living cells of $\mathrm{Ca}^{2+}$-dependent changes in the fluorescence emission of an indicator composed of two green fluorescent protein variants linked by a calmodulin-binding sequence. A new class of fluorescent indicators. J. Biol. Chem. 272, 13270-13274. doi: 10.1074/jbc.272.20.13270

Sakai, R., Repunte-Canonigo, V., Raj, C. D., and Knöpfel, T. (2001). Design and characterization of a DNA-encoded, voltage-sensitive fluorescent protein. Eur. J. Neurosci. 13, 2314-2318. doi: 10.1046/j.0953-816x.2001.01617.x

Sawinski, J., Wallace, D. J., Greenberg, D. S., Grossmann, S., Denk, W., and Kerr, J. N. (2009). Visually evoked activity in cortical cells imaged in freely moving animals. Proc. Natl. Acad. Sci. U.S.A. 106, 19557-19562. doi: 10.1073/pnas.0903680106

Seelig, J. D., Chiappe, M. E., Lott, G. K., Dutta, A., Osborne, J. E., Reiser, M. B., et al. (2010). Two-photon calcium imaging from head-fixed Drosophila during optomotor walking behavior. Nat. Methods 7, 535-540. doi: 10.1038/nmeth.1468

Shimomura, O., Johnson, F. H., and Saiga, Y. (1962). Extraction, purification and properties of aequorin, a bioluminescent protein from the luminous hydromedusan, Aequorea. J. Cell. Comp. Physiol. 59, 223-239. doi: 10.1002/jcp.1030590302

Siegel, M. S., and Isacoff, E. Y. (1997). A genetically encoded optical probe of membrane voltage. Neuron 19, 735-741. doi: 10.1016/S0896-6273(00)80955-1

Singer, J. H., and Diamond, J. S. (2006). Vesicle depletion and synaptic depression at a mammalian ribbon synapse. J. Neurophysiol. 95, 3191-3198. doi: 10.1152/jn.01309.2005

Souslova, E. A., Belousov, V. V., Lock, J. G., Strömblad, S., Kasparov, S., Bolshakov, A. P., et al. (2007). Single fluorescent protein-based $\mathrm{Ca}^{2+}$ sensors with increased dynamic range. BMC Biotechnol. 7:37. doi: 10.1186/1472-6750-7-37

Stosiek, C., Garaschuk, O., Holthoff, K., and Konnerth, A. (2003). In vivo twophoton calcium imaging of neuronal networks. Proc. Natl. Acad. Sci. U.S.A. 100, 7319-7324. doi: 10.1073/pnas. 1232232100
St-Pierre, F., Marshall, J. D., Yang, Y., Gong, Y., Schnitzer, M. J., and Lin, M. Z. (2014). High-fidelity optical reporting of neuronal electrical activity with an ultrafast fluorescent voltage sensor. Nat. Neurosci. 17, 884-889. doi: 10.1038/nn.3709

Sun, X. R., Badura, A., Pacheco, D. A., Lynch, L. A., Schneider, E. R., Taylor, M. P., et al. (2013). Fast GCaMPs for improved tracking of neuronal activity. Nat. Commun. 4, 2170. doi: 10.1038/ncomms3170

Tabata, H., and Nakajima, K. (2001). Efficient in utero gene transfer system to the developing mouse brain using electroporation: visualization of neuronal migration in the developing cortex. Neuroscience 103, 865-872. doi: 10.1016/S0306-4522(01)00016-1

Tallini, Y. N., Ohkura, M., Choi, B. R., Ji, G., Imoto, K., Doran, R., et al. (2006). Imaging cellular signals in the heart in vivo: cardiac expression of the high-signal $\mathrm{Ca}^{2+}$ indicator GCaMP2. Proc. Natl. Acad. Sci. U.S.A. 103, 4753-4758. doi: 10.1073/pnas.0509378103

Tank, D. W., Sugimori, M., Connor, J. A., and Llinás, R. R. (1988). Spatially resolved calcium dynamics of mammalian Purkinje cells in cerebellar slice. Science 242, 773-777. doi: 10.1126/science.2847315

Theer, P., Hasan, M. T., and Denk, W. (2003). Two-photon imaging to a depth of 1000 microm in living brains by use of a Ti:Al2O3 regenerative amplifier. Opt. Lett. 28, 1022-1024. doi: 10.1364/OL.28.001022

Thestrup, T., Litzlbauer, J., Bartholomäus, I., Mues, M., Russo, L., Dana, H., et al. (2014). Optimized ratiometric calcium sensors for functional in vivo imaging of neurons and T lymphocytes. Nat. Methods 11, 175-182. doi: 10.1038/nmeth.2773

Tian, L., Hires, S. A., Mao, T., Huber, D., Chiappe, M. E., Chalasani, S. H., et al. (2009). Imaging neural activity in worms, flies and mice with improved GCaMP calcium indicators. Nat. Methods 6, 875-881. doi: 10.1038/nmeth.1398

Tsien, R. Y. (2005). Building and breeding molecules to spy on cells and tumors. FEBS Lett. 579, 927-932. doi: 10.1016/j.febslet.2004.11.025

Turrigiano, G. G., Leslie, K. R., Desai, N. S., Rutherford, L. C., and Nelson, S. B. (1998). Activity-dependent scaling of quantal amplitude in neocortical neurons. Nature 391, 892-896. doi: 10.1038/36103

Urnov, F. D., Rebar, E. J., Holmes, M. C., Zhang, H. S., and Gregory, P. D. (2010). Genome editing with engineered zinc finger nucleases. Nat. Rev. Genet. 11, 636646. doi: $10.1038 / \mathrm{nrg} 2842$

Valmianski, I., Shih, A. Y., Driscoll, J. D., Matthews, D. W., Freund, Y., and Kleinfeld, D. (2010). Automatic identification of fluorescently labeled brain cells for rapid functional imaging. J. Neurophysiol. 104, 1803-1811. doi: 10.1152/jn.00484.2010

VanEngelenburg, S. B., and Palmer, A. E. (2008). Fluorescent biosensors of protein function. Curr. Opin. Chem. Biol. 12, 60-65. doi: 10.1016/j.cbpa.2008.01.020

Van Keuren, M. L., Gavrilina, G. B., Filipiak, W. E., Zeidler, M. G., and Saunders, T. L. (2009). Generating transgenic mice from bacterial artificial chromosomes: transgenesis efficiency, integration and expression outcomes. Transgenic Res. 18, 769-785. doi: 10.1007/s11248-009-9271-2

Vogelstein, J. T., Packer, A. M., Machado, T. A., Sippy, T., Babadi, B., Yuste, R., et al. (2010). Fast nonnegative deconvolution for spike train inference from population calcium imaging. J. Neurophysiol. 104, 3691-3704. doi: 10.1152/jn.01073.2009

Vogelstein, J. T., Watson, B. O., Packer, A. M., Yuste, R., Jedynak, B., and Paninski, L. (2009). Spike inference from calcium imaging using sequential Monte Carlo methods. Biophys. J. 97, 636-655. doi: 10.1016/j.bpj.2008.08.005

Wallace, D. J., Meyer zum Alten Borgloh, S., Astori, S., Yang, Y., Bausen, M., Kügler, S., et al. (2008). Single-spike detection in vitro and in vivo with a genetic $\mathrm{Ca}^{2+}$ sensor. Nat. Methods 5, 797-804. doi: 10.1038/nmeth.1242

Wang, D., McMahon, S., Zhang, Z., and Jackson, M. B. (2012). Hybrid voltage sensor imaging of electrical activity from neurons in hippocampal slices from transgenic mice. J. Neurophysiol. 108, 3147-3160. doi: 10.1152/jn.00722.2012

Wang, J. W., Wong, A. M., Flores, J., Vosshall, L. B., and Axel, R. (2003). Two-photon calcium imaging reveals an odor-evoked map of activity in the fly brain. Cell 112, 271-282. doi: 10.1016/S0092-8674(03)00004-7

Wardill, T. J., Chen, T. W., Schreiter, E. R., Hasseman, J. P., Tsegaye, G., Fosque, B. F., et al. (2013). A neuron-based screening platform for optimizing genetically-encoded calcium indicators. PLoS ONE 8:e77728. doi: 10.1371/journal.pone.0077728

Wurtz, R. H. (1968). Visual cortex neurons: response to stimuli during rapid eye movements. Science 162, 1148-1150. doi: 10.1126/science.162.3858.1148

Yaksi, E., and Friedrich, R. W. (2006). Reconstruction of firing rate changes across neuronal populations by temporally deconvolved $\mathrm{Ca}^{2+}$ imaging. Nat. Methods 3, 377-383. doi: 10.1038/nmeth874

Yamada, Y., Michikawa, T., Hashimoto, M., Horikawa, K., Nagai, T., Miyawaki, A., et al. (2011). Quantitative comparison of genetically encoded Ca indicators in 
cortical pyramidal cells and cerebellar Purkinje cells. Front. Cell. Neurosci. 5:18. doi: $10.3389 /$ fncel.2011.00018

Yin, L., Masella, B., Dalkara, D., Zhang, J., Flannery, J. G., Schaffer, D. V., et al. (2014). Imaging light responses of foveal ganglion cells in the living macaque eye. J. Neurosci. 34, 6596-6605. doi: 10.1523/JNEUROSCI.4438-13.2014

Zariwala, H. A., Borghuis, B. G., Hoogland, T. M., Madisen, L., Tian, L., De Zeeuw, C. I., et al. (2012). A Cre-dependent GCaMP3 reporter mouse for neuronal imaging in vivo. J. Neurosci. 32, 3131-3141. doi: 10.1523/JNEUROSCI.4469-11.2012

Zeng, H., and Madisen, L. (2012). Mouse transgenic approaches in optogenetics. Prog. Brain Res. 196, 193-213. doi: 10.1016/B978-0-444-59426-6.00010-0

Zhao, Y., Araki, S., Wu, J., Teramoto, T., Chang, Y. F., Nakano, M., et al. (2011). An expanded palette of genetically encoded $\mathrm{Ca}^{2+}$ indicators. Science 333, 1888-1891. doi: 10.1126/science. 1208592

Ziv, Y., Burns, L. D., Cocker, E. D., Hamel, E. O., Ghosh, K. K., Kitch, L. J., et al. (2013). Long-term dynamics of CA1 hippocampal place codes. Nat. Neurosci. 16, 264-266. doi: 10.1038/nn.3329
Conflict of Interest Statement: The authors declare that the research was conducted in the absence of any commercial or financial relationships that could be construed as a potential conflict of interest.

Received: 28 August 2014; accepted: 15 November 2014; published online: 05 December 2014.

Citation: Broussard GJ, Liang R and Tian L (2014) Monitoring activity in neural circuits with genetically encoded indicators. Front. Mol. Neurosci. 7:97. doi: 10.3389/fnmol.2014.00097

This article was submitted to the journal Frontiers in Molecular Neuroscience. Copyright $\odot 2014$ Broussard, Liang and Tian. This is an open-access article distributed under the terms of the Creative Commons Attribution License (CC BY). The use, distribution or reproduction in other forums is permitted, provided the original author(s) or licensor are credited and that the original publication in this journal is cited, in accordance with accepted academic practice. No use, distribution or reproduction is permitted which does not comply with these terms. 